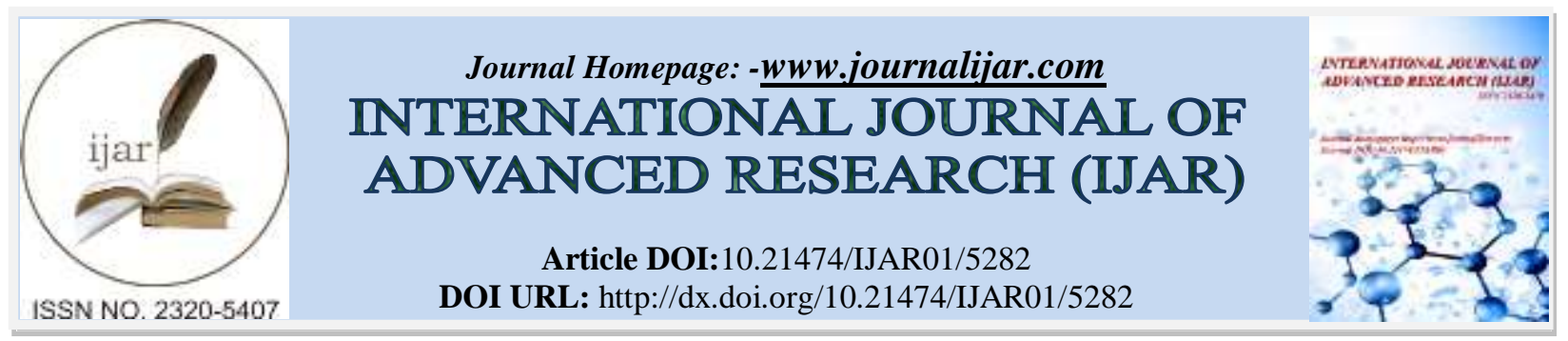

RESEARCH ARTICLE

\title{
SOME CLASSES OF $(\kappa, \mu)$-CONTACT METRIC MANIFOLD SATISFYING SEMISYMMETRIC CONDITIONS.
}

Divyashree G. and Venkatesha.

Department of Mathematics, Kuvempu University,Shankaraghatta - 577 451, Shimoga, Karnataka, INDIA.

\section{Manuscript Info}

Manuscript History

Received: 25 June 2017

Final Accepted: 27 July 2017

Published: August 2017

Key words:-

$(\kappa, \mu)$-contact metric

manifolds, C-Bochner

curvature tensor, $\mathrm{m}$ -

projective curvature tensor,

semisymmetry, non-Sasakian

manifolds, $\eta$-Einstein

manifold.

\section{Abstract}

In the present paper, we consider $C$-Bochner and $m$-projective curvature tensors in a non-Sasakian $(\kappa, \mu)$-contact metric manifold. Moreover, we study the curvature tensors satisfying semisymmetric conditions on the same manifold and obtained results.

Copy Right, IJAR, 2017,. All rights reserved.

\section{Introduction:-}

In 1995, Blair et al.[3] introduced a class of contact metric manifolds for which the characteristic vector field $\xi$ belongs to the $(\kappa, \mu)$-nullity distribution for some real numbers $\kappa$ and $\mu$ and are known as $(\kappa, \mu)$-contact metric manifolds. Boeckx[4] gave a full classification of $(\kappa, \mu)$-contact metric manifolds. The class of $(\kappa, \mu)$-contact metric manifolds encloses both Sasakian and non-Sasakian manifolds. Also non-Sasakian $(\kappa, \mu)$-contact metric manifolds have two classes, namely, the first class consists of the unit tangent sphere bundles of spaces of constant curvature, equipped with their natural contact metric structure and the second class contains all the three-dimensional unimodular Lie groups, except the commutative one, admitting the structure of a left invariant $(\kappa, \mu)$-contact metric manifold $[3,4,17]$. $(\kappa, \mu)$-contact metric manifolds have studied by several authors in the following papers $[1,10$, $11,19]$ and others.

A $(0, q)$-tensor field $T$ on $\left(M^{2 n+1}, g\right)$ is called parallel when it is invariant under parallel translation, i.e., $\nabla T=0$. Among the geometric properties of manifolds, symmetry is an important one. A Riemannian manifold $M^{2 n+1}$ is called locally symmetric if its curvature tensor $R$ is parallel, i.e., $\nabla R=0$, where $\nabla$ denotes the Levi-Civita connection. As a generalization of locally symmetric spaces, many geometers have considered semisymmetric spaces and their generalizations. 
A Riemannian manifold $M^{2 n+1}$ is said to be semisymmetric if its curvature tensor $R$ satisfies

$$
R(X, Y) \cdot R=0
$$

for any vector fields $X, Y \in T_{p} M^{2 n+1}$, where $R(X, Y)$ acts on $R$ as a derivation [13, 20].

In 1969, Matsumoto and Chuman[14] introduced the concept of $C$-Bochner curvature tensor in a Sasakian manifold and studied its several properties. Many authors have studied the characterizations of $C$-Bochner curvature tensor with different manifolds in the papers $[8,9,12,19]$ and others.

The $C$-Bochner curvature tensor is given by

$$
\begin{aligned}
\mathrm{B}(\mathrm{X}, \mathrm{Y}) \mathrm{Z}= & \mathrm{R}(\mathrm{X}, \mathrm{Y}) \mathrm{Z}+\frac{1}{2 \mathrm{n}+4}[\mathrm{~S}(\mathrm{X}, \mathrm{Z}) \mathrm{Y}-\mathrm{S}(\mathrm{Y}, \mathrm{Z}) \mathrm{X}+\mathrm{g}(\mathrm{X}, \mathrm{Z}) \mathrm{QY}-\mathrm{g}(\mathrm{Y}, \mathrm{Z}) \mathrm{QX} \\
& +\mathrm{S}(\phi \mathrm{X}, \mathrm{Z}) \phi \mathrm{Y}-\mathrm{S}(\phi \mathrm{Y}, \mathrm{Z}) \phi \mathrm{X}+g(\phi \mathrm{X}, \mathrm{Z}) \mathrm{Q} \phi \mathrm{Y}-g(\phi \mathrm{Y}, \mathrm{Z}) \mathrm{Q} \phi \mathrm{X} \\
& +2 \mathrm{~S}(\phi \mathrm{X}, \mathrm{Y}) \phi \mathrm{Z}+2 g(\phi \mathrm{X}, \mathrm{Y}) \mathrm{Q} \phi \mathrm{Z}-\mathrm{S}(\mathrm{X}, \mathrm{Z}) \eta(\mathrm{Y}) \xi+\mathrm{S}(\mathrm{Y}, \mathrm{Z}) \eta(\mathrm{X}) \xi \\
& -\eta(\mathrm{X}) \eta(\mathrm{Z}) \mathrm{QY}+\eta(\mathrm{Y}) \eta(\mathrm{Z}) \mathrm{QX}]-\frac{\mathrm{p}+2 \mathrm{n}}{2 \mathrm{n}+4}[g(\phi \mathrm{X}, \mathrm{Z}) \phi \mathrm{Y}-g(\phi \mathrm{Y}, \mathrm{Z}) \phi \mathrm{X} \\
& +2 g(\phi \mathrm{X}, \mathrm{Y}) \phi \mathrm{Z}]-\frac{\mathrm{p}-4}{2 \mathrm{n}+4}[g(\mathrm{X}, \mathrm{Z}) \mathrm{Y}-g(\mathrm{Y}, \mathrm{Z}) \mathrm{X}]+\frac{\mathrm{p}}{2 \mathrm{n}+4}[g(\mathrm{X}, \mathrm{Z}) \eta(\mathrm{Y}) \xi \\
& -g(\mathrm{Y}, \mathrm{Z}) \eta(\mathrm{X}) \xi+\eta(\mathrm{X}) \eta(\mathrm{Z}) \mathrm{Y}-\eta(\mathrm{Y}) \eta(\mathrm{Z}) \mathrm{X}],
\end{aligned}
$$

where $S$ is the Ricci tensor of type (0,2), $Q$ is the Ricci operator defined by $g(Q X, Y)=S(X, Y), p=\frac{r+2 n}{2 n+2}$ and $r$ is the scalar curvature of the manifold.

In 1971, Pokhariyal and Mishra [18] defined a tensor field $W^{*}$ on a Riemannian manifold as

$$
W^{*}(X, Y) Z=R(X, Y) Z-\frac{1}{4 n}[S(Y, Z) X-S(X, Z) Y+g(Y, Z) Q X-g(X, Z) Q Y] .
$$

Such a tensor field $W^{*}$ is known as $m$-projective curvature tensor. Ojha[15, 16] studied the properties of the $m$ projective curvature tensor in Sasakian and Kaehler manifold. The properties of $m$-projective curvature tensor were studied on different manifolds in $[6,7,23]$ and others.

The present paper is organized in the following way: Section 2 is concerned with preliminaries of $(\kappa, \mu)$-contact metric manifold. We study $h$-C-Bochner semisymmetric, $\phi$-C-Bochner semisymmetric, $h$ - $m$-projectively semisymmetric, $\phi$ - $m$-projectively semisymmetric non-Sasakian $(\kappa, \mu)$-contact metric manifolds in sections $3,4,5$ and 6 , respectively. In all the cases the manifold appears to be an $\eta$-Einstein manifold.

\section{Preliminaries:-}

A $(2 n+1)$-dimensional differentiable manifold $M$ is called a contact manifold [2] if it carries a global 1-form $\eta$ such that $\eta \wedge(d \eta)^{2 n+1} \neq 0$ everywhere on $M$. It is well known that a contact metric manifold admits an almost contact metric structure $(\phi, \xi, \eta, g)$, i.e., a global vector field $\xi$, which is called the characteristic vector field, a $(1,1)$-tensor field $\phi$ and a Riemannian metric $g$ such that

$$
\begin{aligned}
\phi^{2} & =-\mathrm{I}+\eta \otimes \xi, \mathrm{g}(\mathrm{X}, \xi)=\eta(\mathrm{X}), \\
\eta(\xi) & =1, g(X, Y)=g(\phi X, \phi Y)+\eta(X) \eta(Y) .
\end{aligned}
$$

Furthermore, $(\phi, \xi, \eta, g)$ can be chosen such that

$$
d \eta(X . Y)=g(X, \phi Y), g(X, \phi Y)=-g(Y, \phi X),
$$


for all vector fields $X, Y \in T_{p} M^{2 n+1}$ and we call such a structure as contact metric structure. A manifold $M$ with such a structure is said to be contact metric manifold and it is denoted by $M(\phi, \xi, \eta, g)$. Along with the above relations, we have

$$
\phi \xi=0, \eta \circ \phi=0, d \eta(\xi, X)=0 .
$$

We define a $(1,1)$-tensor field $h$ by $h=\frac{1}{2} £_{\xi} \phi$, where $€_{\xi}$ is the Lie differentiation in the direction of $\xi$. Since tensor field $h$ is self-adjoint and anticommutes with $\phi$, therefore

$$
h \xi=0, \phi h+h \phi=0, \operatorname{trh}=\operatorname{tr} \phi h=0, \nabla_{X} \xi=-\phi X-\phi h X,
$$

where $\nabla$ is the Levi-Civita connection and if $X \neq 0$ is an eigenvector of $h$ corresponding to the eigenvalue $\lambda$, then $\phi X$ is an eigenvector of $h$ corresponding to the eigenvalue $-\lambda$. Blair et al. [3] considered and studied the $(\kappa, \mu)$ nullity condition. The $(\kappa, \mu)$-nullity distribution $N(\kappa, \mu)$ [3] of a contact metric manifold $M$ is defined by $(\kappa, \mu)$ : $p \rightarrow N_{p}(\kappa, \mu)=\left[Z \in T_{p} M^{2 n+1}: R(X, Y) Z=(\kappa I+\mu h)\{g(Y, Z) X-g(X, Z) Y\}\right]$ for all $X, Y \in T_{p} M^{2 n+1}$. A contact metric manifold $M^{2 n+1}$ with $\xi \in N(\kappa, \mu)$ is called a $(\kappa, \mu)$-contact metric manifold and we have

$$
R(X, Y) \xi=\kappa\{\eta(Y) X-\eta(X) Y\}+\mu\{\eta(Y) h X-\eta(X) h Y\},
$$

for all $X, Y \in T_{p} M^{2 n+1}$. If $\mu=0$, then the $(\kappa, \mu)$-nullity distribution $N(\kappa, \mu)$ is reduced to $\kappa$-nullity distribution $N(\kappa)$ [22]. If $\xi \in N(\kappa)$, then we call contact metric manifold $M$ an $N(\kappa)$-contact metric manifold. The class of $(\kappa, \mu)$-contact metric manifolds contains both classes of Sasakian $\{(k=1)$ and $(h=0)\}$ and non-Sasakian $\{(k \neq 1)$ and $(h \neq 0)\}$ manifolds. In a $(\kappa, \mu)$-contact metric manifold, the following relations hold:

$$
\begin{aligned}
h^{2}= & (\kappa-1) \phi^{2}, \\
R(\xi, X) Y= & \kappa[g(X, Y) \xi-\eta(Y) X]+\mu[g(h X, Y) \xi-\eta(Y) h X], \\
S(X, \xi)= & 2 n \kappa \eta(X), \\
S(X, Y)= & {[2(n-1)-n \mu] g(X, Y)+[2(n-1)+\mu] g(h X, Y) } \\
& +[2(1-n)+n(2 \kappa+\mu)] \eta(X) \eta(Y), \\
S(\phi X, \phi Y)= & S(X, Y)-2 n k \eta(X) \eta(Y)-2(2 n-2+\mu) g(h X, Y), \\
g(Q X, Y)= & S(X, Y),
\end{aligned}
$$

where $S$ is the Ricci tensor of type $(0,2), Q$ is the Ricci operator and $r$ is the scalar curvature of the manifold.

$$
Q \phi-\phi Q=2[2(n-1)+\mu] h \phi .
$$

In general, in a $(\kappa, \mu)$-contact metric manifold $Q$ does not commutes with $\phi$. However, Yildiz and De [1] proved the following:

Proposition 2.1.[1] In a non-Sasakian $(\kappa, \mu)$-contact metric manifold the following conditions are equivalent:

(i) $M$ is an $\eta$-Einstein manifold,

(ii) $Q \phi=\phi Q$ in $M$.

Corollary 2.1.[1] A 3-dimensional non-Sasakian $(\kappa, \mu)$-contact $\eta$-Einstein manifold is an $N(k)$-contact metric manifold.

The following lemma which was proved in [3] is helpful to state our theorem.

Lemma 2.1.[3]: Let $M^{2 n+1}(\phi, \xi, \eta, g)$ be a contact metric manifold with $\xi$ belonging to the $(\kappa, \mu)$-nullity distribution. Then for any vector fields $X, Y, Z$, we have 


$$
\begin{aligned}
\mathrm{R}(\mathrm{X}, \mathrm{Y}) h \mathrm{Z}-\mathrm{hR}(\mathrm{X}, \mathrm{Y}) \mathrm{Z}= & \{\kappa[g(h \mathrm{Y}, \mathrm{Z}) \eta(\mathrm{X})-g(h \mathrm{X}, \mathrm{Z}) \eta(\mathrm{Y})]+\mu(\kappa-1)[g(\mathrm{X}, \mathrm{Z}) \eta(\mathrm{Y}) \\
& -g(\mathrm{Y}, \mathrm{Z}) \eta(\mathrm{X})]\} \xi+\kappa\{g(\mathrm{Y}, \phi \mathrm{Z}) \phi h \mathrm{X}-g(\mathrm{X}, \phi \mathrm{Z}) \phi h \mathrm{Y} \\
& +g(\mathrm{Z}, \phi h \mathrm{Y}) \phi \mathrm{X}-g(\mathrm{Z}, \phi h \mathrm{X}) \phi \mathrm{Y}+\eta(\mathrm{Z})[\eta(\mathrm{X}) h \mathrm{Y}-\eta(\mathrm{Y}) h \mathrm{X}]\} \\
& -\mu\{\eta(\mathrm{Y})[(1-\kappa) \eta(\mathrm{Z}) \mathrm{X}+\mu \eta(\mathrm{X}) h \mathrm{Z}]-\eta(\mathrm{X})[(1-\kappa) \eta(\mathrm{Z}) \mathrm{Y} \\
& +\mu \eta(\mathrm{Y}) h \mathrm{Z}]+2 \mathrm{~g}(\mathrm{X}, \phi \mathrm{Y}) \phi h \mathrm{Z} .
\end{aligned}
$$

Lemma 2.2.[3]: Let $M^{2 n+1}(\phi, \xi, \eta, g)$ be a contact metric manifold with $\xi$ belonging to the $(\kappa, \mu)$-nullity distribution. Then for any vector fields $X, Y, Z$, we have

$$
\begin{aligned}
\mathrm{R}(\mathrm{X}, \mathrm{Y}) \phi \mathrm{Z}-\phi \mathrm{R}(\mathrm{X}, \mathrm{Y}) \mathrm{Z}= & \{(1-\kappa)[g(\phi \mathrm{Y}, \mathrm{Z}) \eta(\mathrm{X})-g(\phi \mathrm{X}, \mathrm{Z}) \eta(\mathrm{Y})] \\
& +(1-\mu)[g(\phi h \mathrm{Y}, \mathrm{Z}) \eta(\mathrm{X})-g(\phi h \mathrm{X}, \mathrm{Z})(\mathrm{Y})]\} \xi \\
& -g(\mathrm{Y}+h \mathrm{Y}, \mathrm{Z})(\phi \mathrm{X}+\phi h \mathrm{X})+g(\mathrm{X}+h \mathrm{X}, \mathrm{Z})(\phi \mathrm{Y}+\phi h \mathrm{Y}) \\
& -g(\phi \mathrm{Y}+\phi h \mathrm{Y}, \mathrm{Z})(\mathrm{X}+h \mathrm{X})+g(\phi \mathrm{X}+\phi h \mathrm{X}, \mathrm{Z})(\mathrm{Y}+h \mathrm{Y}) \\
& -\eta(\mathrm{Z})\{(1-\kappa)[\eta(\mathrm{X}) \phi \mathrm{Y}-\eta(\mathrm{Y}) \phi \mathrm{X}]+(1-\mu)[\eta(\mathrm{X}) \phi h \mathrm{Y} \\
& -\eta(\mathrm{Y}) \phi h \mathrm{X})]\} .
\end{aligned}
$$

3. $h$ - $C$-Bochner semisymmetric non-Sasakian $(\kappa, \mu)$-contact metric manifold

Definition 3.1. A Riemannian manifold $\left(\mathrm{M}^{2 n+1}, g\right)$ is said to be $h-C$-Bochner semisymmetric if

$$
\mathrm{B}(\mathrm{X}, \mathrm{Y}) \cdot h=0 \text {, }
$$

holds on $M$.

Let $\mathrm{M}^{2 n+1}$ be a $h$-C -Bochner semisymmetric non-Sasakian $(\kappa, \mu)$-contact metric manifold. The condition $B(X, Y)$. $h=0$ can be expressed as follows,

$$
(\mathrm{B}(\mathrm{X}, \mathrm{Y}) \cdot h) \mathrm{Z}=\mathrm{B}(\mathrm{X}, \mathrm{Y}) h \mathrm{Z}-h \mathrm{~B}(\mathrm{X}, \mathrm{Y}) \mathrm{Z}=0,
$$

for any vector fields $X, Y, Z$.

By using (1.1) and (2.14) in (3.2), we have

$$
\begin{aligned}
& \{\kappa[g(h \mathrm{Y}, \mathrm{Z}) \eta(\mathrm{X})-g(h \mathrm{X}, \mathrm{Z}) \eta(\mathrm{Y})]+\mu(\kappa-1)[g(\mathrm{X}, \mathrm{Z}) \eta(\mathrm{Y})-g(\mathrm{Y}, \mathrm{Z}) \eta(\mathrm{X})]\} \xi \\
& +\kappa\{g(\mathrm{Y}, \phi \mathrm{Z}) \phi h \mathrm{X}-g(\mathrm{X}, \phi \mathrm{Z}) \phi h \mathrm{Y}+g(\mathrm{Z}, \phi h \mathrm{Y}) \phi \mathrm{X}-g(\mathrm{Z}, \phi h \mathrm{X}) \phi \mathrm{Y}+\eta(\mathrm{Z})[\eta(\mathrm{X}) h \mathrm{Y} \\
& -\eta(\mathrm{Y}) h \mathrm{X}]\}-\mu\{\eta(\mathrm{Y})[(1-\kappa) \eta(\mathrm{Z}) \mathrm{X}+\mu \eta(\mathrm{X}) h \mathrm{Z}]-\eta(\mathrm{X})[(1-\kappa) \eta(\mathrm{Z}) \mathrm{Y}+\mu \eta(\mathrm{Y}) h \mathrm{Z}] \\
& +2 g(\mathrm{X}, \phi \mathrm{Y}) \phi h \mathrm{Z}\}+\frac{1}{2 \mathrm{n}+4}[\mathrm{~S}(\mathrm{X}, h \mathrm{Z}) \mathrm{Y}-\mathrm{S}(\mathrm{Y}, h \mathrm{Z}) \mathrm{X}+g(\mathrm{X}, h \mathrm{Z}) \mathrm{QY}-g(\mathrm{Y}, h \mathrm{Z}) \mathrm{QX} \\
& +\mathrm{S}(\phi \mathrm{X}, h \mathrm{Z}) \phi \mathrm{Y}-\mathrm{S}(\phi \mathrm{Y}, h \mathrm{Z}) \phi \mathrm{X}+g(\phi \mathrm{X}, h \mathrm{Z}) \mathrm{Q} \phi \mathrm{Y}-g(\phi \mathrm{Y}, h \mathrm{Z}) \mathrm{Q} \phi \mathrm{X}+2 \mathrm{~S}(\phi \mathrm{X}, \mathrm{Y}) \phi h \mathrm{Z} \\
& +2 g(\phi \mathrm{X}, \mathrm{Y}) \mathrm{Q} \phi h \mathrm{Z}-\mathrm{S}(\mathrm{X}, h \mathrm{Z}) \eta(\mathrm{Y}) \xi+\mathrm{S}(\mathrm{Y}, h \mathrm{Z}) \eta(\mathrm{X}) \xi-\mathrm{S}(\mathrm{X}, \mathrm{Z}) h \mathrm{Y}+\mathrm{S}(\mathrm{Y}, \mathrm{Z}) h \mathrm{X}-g(\mathrm{X}, \mathrm{Z}) h \mathrm{QY} \\
& +g(\mathrm{Y}, \mathrm{Z}) h \mathrm{QX}-\mathrm{S}(\phi \mathrm{X}, \mathrm{Z}) h \phi \mathrm{Y}+\mathrm{S}(\phi \mathrm{Y}, \mathrm{Z}) h \phi \mathrm{X}-g(\phi \mathrm{X}, \mathrm{Z}) h \mathrm{Q} \phi \mathrm{Y}+g(\phi \mathrm{Y}, \mathrm{Z}) h \mathrm{Q} \phi \mathrm{X}
\end{aligned}
$$




$$
\begin{aligned}
& -2 \mathrm{~S}(\phi \mathrm{X}, \mathrm{Y}) h \phi \mathrm{Z}-2 g(\phi \mathrm{X}, \mathrm{Y}) h \mathrm{Q} \phi \mathrm{Z}+\eta(\mathrm{X}) \eta(\mathrm{Z}) h \mathrm{QY}-\eta(\mathrm{Y}) \eta(\mathrm{Z}) h \mathrm{QX}]-\frac{\mathrm{p}+2 \mathrm{n}}{2 \mathrm{n}+4}[g(\phi \mathrm{X}, h \mathrm{Z}) \phi \mathrm{Y} \\
& -g(\phi \mathrm{Y}, h \mathrm{Z}) \phi \mathrm{X}+2 g(\phi \mathrm{X}, \mathrm{Y}) h \phi \mathrm{Z}-g(\phi \mathrm{X}, \mathrm{Z}) h \phi \mathrm{Y}+g(\phi \mathrm{Y}, \mathrm{Z}) h \phi \mathrm{X}-2 g(\phi \mathrm{X}, \mathrm{Y}) h \phi \mathrm{Z}] \\
& -\frac{\mathrm{p}-4}{2 \mathrm{n}+4}[g(\mathrm{X}, h \mathrm{Z}) \mathrm{Y}-g(\mathrm{Y}, h \mathrm{Z}) \mathrm{X}-g(\mathrm{X}, \mathrm{Z}) h \mathrm{Y}+g(\mathrm{Y}, \mathrm{Z}) h \mathrm{X}]+\frac{\mathrm{p}}{2 \mathrm{n}+4}[g(\mathrm{X}, h \mathrm{Z}) \eta(\mathrm{Y}) \xi \\
& -g(\mathrm{Y}, h \mathrm{Z}) \eta(\mathrm{X}) \xi-\eta(\mathrm{X}) \eta(\mathrm{Z}) h \mathrm{Y}+\eta(\mathrm{Y}) \eta(\mathrm{Z}) h \mathrm{X}]=0 .
\end{aligned}
$$

Substituting $X$ by $h X$ in (3.3) and using (2.1), (2.4) and (2.7), we obtain

$$
\begin{aligned}
& \kappa(\kappa-1) g(\mathrm{X}, \mathrm{Z}) \eta(\mathrm{Y}) \xi-\kappa(\kappa-1) \eta(\mathrm{X}) \eta(\mathrm{Y}) \eta(\mathrm{Z}) \xi+\mu(\kappa-1) g(h \mathrm{X}, \mathrm{Z}) \eta(\mathrm{Y}) \xi \\
& -\kappa(\kappa-1) g(\mathrm{Y}, \phi \mathrm{Z}) \phi \mathrm{X}-\kappa g(h \mathrm{X}, \phi \mathrm{Z}) \phi h \mathrm{Y}+\kappa g(\mathrm{Z}, \phi h \mathrm{Y}) \phi h \mathrm{X}+\kappa(\kappa-1) g(\mathrm{Z}, \phi \mathrm{X}) \phi \mathrm{Y} \\
& +\kappa(\kappa-1) \eta(\mathrm{Y}) \eta(\mathrm{Z}) \mathrm{X}-\kappa(\kappa-1) \eta(\mathrm{X}) \eta(\mathrm{Y}) \eta(\mathrm{Z}) \xi-\mu(1-\kappa) \eta(\mathrm{Y}) \eta(\mathrm{Z}) h \mathrm{X}-2 \mu g(h \mathrm{X}, \phi \mathrm{Y}) h \mathrm{Z} \\
& \frac{1}{2 \mathrm{n}+4}[\mathrm{~S}(h \mathrm{X}, h \mathrm{Z}) \mathrm{Y}-\mathrm{S}(\mathrm{Y}, h \mathrm{Z}) h \mathrm{X}-(\kappa-1) g(\mathrm{X}, \mathrm{Z}) \mathrm{QY}+(\kappa-1) \eta(\mathrm{X}) \eta(\mathrm{Z}) \mathrm{QY}-g(\mathrm{Y}, h \mathrm{Z}) \mathrm{Q} h \mathrm{X} \\
& +\mathrm{S}(\phi h \mathrm{X}, h \mathrm{Z}) \phi \mathrm{Y}-\mathrm{S}(\phi \mathrm{Y}, h \mathrm{Z}) \phi h \mathrm{X}+g(\phi h \mathrm{X}, h \mathrm{Z}) \mathrm{Q} \phi \mathrm{Y}-g(\phi \mathrm{Y}, h \mathrm{Z}) \mathrm{Q} \phi h \mathrm{X}+2 \mathrm{~S}(\phi h \mathrm{X}, \mathrm{Y}) \phi h \mathrm{Z} \\
& +2 g(\phi h \mathrm{X}, \mathrm{Y}) \mathrm{Q} \phi h \mathrm{Z}-\mathrm{S}(h \mathrm{X}, h \mathrm{Z}) \eta(\mathrm{Y}) \xi-\mathrm{S}(h \mathrm{X}, \mathrm{Z}) h \mathrm{Y}-(\kappa-1) \mathrm{S}(\mathrm{Y}, \mathrm{Z}) \mathrm{X}+(\kappa-1) \mathrm{S}(\mathrm{Y}, \mathrm{Z}) \eta(\mathrm{X}) \xi \\
& -g(h \mathrm{X}, \mathrm{Z}) h \mathrm{QY}-(\kappa-1) g(\mathrm{Y}, \mathrm{Z}) \mathrm{QX}+(\kappa-1) g(\mathrm{Y}, \mathrm{Z}) \eta(\mathrm{X}) \mathrm{Q} \xi-\mathrm{S}(\phi h \mathrm{X}, \mathrm{Z}) h \phi \mathrm{Y} \\
& +(\kappa-1) \mathrm{S}(\phi \mathrm{Y}, \mathrm{Z}) \phi \mathrm{X}-g(\phi h \mathrm{X}, \mathrm{Z}) h \mathrm{Q} \phi \mathrm{Y}+(\kappa-1) g(\phi \mathrm{Y}, \mathrm{Z}) \mathrm{Q} \phi \mathrm{X}-2 \mathrm{~S}(\phi h \mathrm{X}, \mathrm{Y}) h \phi \mathrm{Z} \\
& -2 g(\phi h \mathrm{X}, \mathrm{Y}) h \mathrm{Q} \phi \mathrm{Z}+(\kappa-1) \eta(\mathrm{Y}) \eta(\mathrm{Z}) \mathrm{QX}-(\kappa-1) \eta(\mathrm{X}) \eta(\mathrm{Y}) \eta(\mathrm{Z}) \mathrm{Q} \xi]-\frac{\mathrm{p}+2 \mathrm{n}}{2 \mathrm{n}+4}[g(\phi h \mathrm{X}, h \mathrm{Z}) \phi \mathrm{Y} \\
& -g(\phi \mathrm{Y}, h \mathrm{Z}) \phi h \mathrm{X}+2 g(\phi h \mathrm{X}, \mathrm{Y}) \phi h \mathrm{Z}-g(\phi h \mathrm{X}, \mathrm{Z}) h \phi \mathrm{Y}+(\kappa-1) g(\phi \mathrm{Y}, \mathrm{Z}) \mathrm{X}-2 g(\phi h \mathrm{X}, \mathrm{Y}) h \phi \mathrm{Z}] \\
& -\frac{\mathrm{p}-4}{2 \mathrm{n}+4}[-(\kappa-1) g(\mathrm{X}, \mathrm{Z}) \mathrm{Y}+(\kappa-1) \eta(\mathrm{X}) \eta(\mathrm{Z}) \mathrm{Y}-g(\mathrm{Y}, h \mathrm{Z}) h \mathrm{X}-g(h \mathrm{X}, \mathrm{Z}) h \mathrm{Y} \\
& -(\kappa-1) g(\mathrm{Y}, \mathrm{Z}) \mathrm{X}+(\kappa-1) g(\mathrm{Y}, \mathrm{Z}) \eta(\mathrm{X}) \xi]+\frac{\mathrm{p}}{2 \mathrm{n}+4}[-(\kappa-1) g(\mathrm{X}, \mathrm{Z}) \eta(\mathrm{Y}) \xi \\
& +(\kappa-1) \eta(\mathrm{Y}) \eta(\mathrm{Z}) \mathrm{X}]=0 .
\end{aligned}
$$

Now taking inner product of (3.4) with $T$ and using symmetry property of $h,(2.9)$ and (2.12), we get

$$
\begin{aligned}
& \kappa(\kappa-1) g(\mathrm{X}, \mathrm{Z}) \eta(\mathrm{Y}) \eta(\mathrm{T})-\kappa(\kappa-1) \eta(\mathrm{X}) \eta(\mathrm{Y}) \eta(\mathrm{Z}) \eta(\mathrm{T})+\mu(\kappa-1) g(h \mathrm{X}, \mathrm{Z}) \eta(\mathrm{Y}) \eta(\mathrm{T}) \\
& -\kappa(\kappa-1) g(\mathrm{Y}, \phi \mathrm{Z}) g(\phi \mathrm{X}, \mathrm{T})-\kappa g(h \mathrm{X}, \phi \mathrm{Z}) g(\phi h \mathrm{Y}, \mathrm{T})+\kappa g(\mathrm{Z}, \phi h \mathrm{Y}) g(\phi h \mathrm{X}, \mathrm{T}) \\
& +\kappa(\kappa-1) g(\mathrm{Z}, \phi \mathrm{X}) g(\phi \mathrm{Y}, \mathrm{T})+\kappa(\kappa-1) g(\mathrm{X}, \mathrm{T}) \eta(\mathrm{Y}) \eta(\mathrm{Z})-\kappa(\kappa-1) \eta(\mathrm{X}) \eta(\mathrm{Y}) \eta(\mathrm{Z}) \eta(\mathrm{T}) \\
& -\mu(1-\kappa) g(h \mathrm{X}, \mathrm{T}) \eta(\mathrm{Y})(\mathrm{Z})-2 \mu g(h \mathrm{X}, \phi \mathrm{Y}) g(\phi h \mathrm{Z}, \mathrm{T})+\frac{1}{2 \mathrm{n}+4}[\mathrm{~S}(h \mathrm{X}, h \mathrm{Z}) g(\mathrm{Y}, \mathrm{T}) \\
& -\mathrm{S}(\mathrm{Y}, h \mathrm{Z}) g(\mathrm{hX}, \mathrm{T})-(\kappa-1) g(\mathrm{X}, \mathrm{Z}) \mathrm{S}(\mathrm{Y}, \mathrm{T})-(\kappa-1) \mathrm{S}(\mathrm{Y}, \mathrm{T}) \eta(\mathrm{X}) \eta(\mathrm{Z})-\mathrm{S}(h \mathrm{X}, \mathrm{T}) g(\mathrm{Y}, h \mathrm{Z})
\end{aligned}
$$




$$
\begin{aligned}
& +\mathrm{S}(\phi h \mathrm{X}, h \mathrm{Z}) g(\phi \mathrm{Y}, \mathrm{T})+\mathrm{S}(\phi \mathrm{Y}, h \mathrm{Z}) g(\phi h \mathrm{X}, \mathrm{T})+g(\phi h \mathrm{X}, h \mathrm{Z}) \mathrm{S}(\phi \mathrm{Y}, \mathrm{T})-g(\phi \mathrm{Y}, h \mathrm{Z}) \mathrm{S}(\phi h \mathrm{X}, \mathrm{T}) \\
& +2 \mathrm{~S}(\phi h \mathrm{X}, \mathrm{Y}) g(\phi h \mathrm{Z}, \mathrm{T})+2 g(\phi h \mathrm{X}, \mathrm{Y}) \mathrm{S}(\phi h \mathrm{Z}, \mathrm{T})-\mathrm{S}(h \mathrm{X}, h \mathrm{Z}) \eta(\mathrm{Y}) \eta(\mathrm{T})-\mathrm{S}(h \mathrm{X}, \mathrm{Z}) g(h \mathrm{Y}, \mathrm{T}) \\
& -(\kappa-1) \mathrm{S}(\mathrm{Y}, \mathrm{Z}) g(\mathrm{X}, \mathrm{T})+(\kappa-1) \mathrm{S}(\mathrm{Y}, \mathrm{Z}) \eta(\mathrm{X}) \eta(\mathrm{T})-g(h \mathrm{X}, \mathrm{Z}) \mathrm{S}(h \mathrm{Y}, \mathrm{T})-(\kappa-1) g(\mathrm{Y}, \mathrm{Z}) \mathrm{S}(\mathrm{X}, \mathrm{T}) \\
& +\kappa(\kappa-1) 2 \mathrm{n} g(\mathrm{Y}, \mathrm{Z}) \eta(\mathrm{X}) \eta(\mathrm{T})-\mathrm{S}(\phi h \mathrm{X}, \mathrm{Z}) g(h \phi \mathrm{Y}, \mathrm{T})+(\kappa-1) \mathrm{S}(\phi \mathrm{Y}, \mathrm{Z}) g(\phi \mathrm{X}, \mathrm{T}) \\
& -g(\phi h \mathrm{X}, \mathrm{Z}) \mathrm{S}(h \phi \mathrm{Y}, \mathrm{T})+(\kappa-1) g(\phi \mathrm{Y}, \mathrm{Z}) \mathrm{S}(\phi \mathrm{X}, \mathrm{T})-2 \mathrm{~S}(\phi h \mathrm{X}, \mathrm{Y}) g(h \phi \mathrm{Z}, \mathrm{T}) \\
& -2 g(\phi h \mathrm{X}, \mathrm{Y}) \mathrm{S}(h \phi \mathrm{Z}, \mathrm{T})+(\kappa-1) \eta(\mathrm{Y}) \eta(\mathrm{Z}) \mathrm{S}(\mathrm{X}, \mathrm{T})-\kappa(\kappa-1) 2 \mathrm{n} \eta(\mathrm{X}) \eta(\mathrm{Y}) \eta(\mathrm{Z}) \eta(\mathrm{T})] \\
& -\frac{\mathrm{p}+2 \mathrm{n}}{2 \mathrm{n}+4}[g(\phi h \mathrm{X}, h \mathrm{Z}) g(\phi \mathrm{Y}, \mathrm{T})-g(\phi \mathrm{Y}, h \mathrm{Z}) g(\phi h \mathrm{X}, \mathrm{T})+2 g(\phi h \mathrm{X}, \mathrm{Y}) g(\phi h \mathrm{Z}, \mathrm{T}) \\
& -g(\phi h \mathrm{X}, \mathrm{Z}) g(h \phi \mathrm{Y}, \mathrm{T})+(\kappa-1) g(\phi \mathrm{Y}, \mathrm{Z}) g(\mathrm{X}, \mathrm{T})-2 g(\phi h \mathrm{X}, \mathrm{Y}) g(h \phi \mathrm{Z}, \mathrm{T})]-\frac{\mathrm{p}-4}{2 \mathrm{n}+4} \\
& {[-(\kappa-1) g(\mathrm{X}, \mathrm{Z}) g(\mathrm{Y}, \mathrm{T})+(\kappa-1) g(\mathrm{Y}, \mathrm{T}) \eta(\mathrm{X}) \eta(\mathrm{Z})-g(\mathrm{Y}, h \mathrm{Z}) g(h \mathrm{X}, \mathrm{T})-g(h \mathrm{X}, \mathrm{Z}) g(h \mathrm{Y}, \mathrm{T})} \\
& -(\kappa-1) g(\mathrm{Y}, \mathrm{Z}) g(\mathrm{X}, \mathrm{T})+(\kappa-1) g(\mathrm{Y}, \mathrm{Z}) \eta(\mathrm{X}) \eta(\mathrm{T})]+\frac{\mathrm{p}}{2 \mathrm{n}+4}[-(\kappa-1) g(\mathrm{X}, \mathrm{Z}) \eta(\mathrm{Y}) \eta(\mathrm{T}) \\
& +(\kappa-1) g(\mathrm{X}, \mathrm{T}) \eta(\mathrm{Y}) \eta(\mathrm{Z})]=0 .
\end{aligned}
$$

Setting $Y=T=\xi$ in (3.5) and using (2.4) and (2.5), we have

$$
\frac{(4 \kappa-1)(\kappa-1)}{2 n+4} g(X, Z)+\frac{(4 \kappa-1)(1-\kappa)}{2 n+4} \eta(X) \eta(Z)+\mu(\kappa-1) g(h X, Z)=0 .
$$

By virtue of (2.10), (3.6) yields to

$$
S(X, Z)=A_{1} g(X, Z)+A_{2} \eta(X) \eta(Z)
$$

where

$A_{1}=\frac{\mu(\kappa-1)(2 n+4)\{2(n-1)-n \mu\}-(4 \kappa-1)(\kappa-1)\{2(n-1)+\mu\}}{\mu(\kappa-1)(2 n+4)}$ and $\quad \mathrm{A}_{2}=\frac{\mu(\kappa-1)(2 \mathrm{n}+4)\{2(1-\mathrm{n})-\mathrm{n}(2 \kappa+\mu)\}-4(\kappa-1)(1-\kappa)\{2(\mathrm{n}-1)+\mu\}}{\mu(\kappa-1)(2 \mathrm{n}+4)}$

Thus we can state the theorem as follows:

Theorem 3.2. Let $M^{2 n+1}(\phi, \xi, \eta, g)$ be a non-Sasakian $(\kappa, \mu)$-contact metric manifold. If $M$ is $h$ - $C$-Bochner semisymmetric then it is an $\eta$-Einstein manifold with constant coefficients.

It follows that from proposition 2.1 and Theorem 3.2 we can state the following:

Corollary 3.2. If $M^{2 n+1}$ be a $h$-C-Bochner semisymmetric $(\kappa, \mu)$-contact metric manifold then the Ricci operator $Q$ commutes with $\phi$ i.e., $Q \phi=\phi Q$.

\section{4. $\phi$ - $C$-Bochner semisymmetric non-Sasakian $(\kappa, \mu)$-contact metric manifold}

Definition 4.2. A Riemannian manifold $\left(\mathrm{M}^{2 n+1}, g\right)$ is said to be $\phi$ - $C$-Bochner semisymmetric if

$$
B(X, Y) \cdot \phi=0,
$$

holds on $M$.

Suppose that $M^{2 n+1}$ is a $\phi$-C-Bochner semisymmetric non-Sasakian $(\kappa, \mu)$-contact metric manifold. The condition $\mathrm{B}(\mathrm{X}, \mathrm{Y}) \cdot \phi=0$ turns into, 


$$
(\mathrm{B}(\mathrm{X}, \mathrm{Y}) \cdot \phi) \mathrm{Z}=\mathrm{B}(\mathrm{X}, \mathrm{Y}) \phi \mathrm{Z}-\phi \mathrm{B}(\mathrm{X}, \mathrm{Y}) \mathrm{Z}=0,
$$

for any vector fields $X, Y, Z$.

Using (1.1) and (2.15) in (4.2), we get

$$
\begin{aligned}
& \{(1-\kappa)[g(\phi \mathrm{Y}, \mathrm{Z}) \eta(\mathrm{X})-g(\phi \mathrm{X}, \mathrm{Z}) \eta(\mathrm{Y})]+(1-\mu)[g(\phi h \mathrm{Y}, \mathrm{Z}) \eta(\mathrm{X})-g(\phi h \mathrm{X}, \mathrm{Z}) \eta(\mathrm{Y})]\} \xi \\
& -g(\mathrm{Y}+h \mathrm{Y})(\phi \mathrm{X}+\phi h \mathrm{X})+g(\mathrm{X}+h \mathrm{X}, \mathrm{Z})(\phi \mathrm{Y}+\phi h \mathrm{Y})-g(\phi \mathrm{Y}+\phi h \mathrm{Y}, \mathrm{Z})(\mathrm{X}+h \mathrm{X}) \\
& +g(\phi \mathrm{X}+\phi h \mathrm{X}, \mathrm{Z})(\mathrm{Y}+h \mathrm{Y})-\eta(\mathrm{Z})\{(1-\kappa)[\eta(\mathrm{X}) \phi \mathrm{Y}-\eta(\mathrm{Y}) \phi \mathrm{X}]+(1-\mu)[\eta(\mathrm{X}) \phi h \mathrm{Y} \\
& -\eta(\mathrm{Y}) \phi h \mathrm{X}]\}+\frac{1}{2 \mathrm{n}+4}[\mathrm{~S}(\mathrm{X}, \phi \mathrm{Z}) \mathrm{Y}-\mathrm{S}(\mathrm{Y}, \phi \mathrm{Z}) \mathrm{X}+g(\mathrm{X}, \phi \mathrm{Z}) \mathrm{QY}-\mathrm{g}(\mathrm{Y}, \phi \mathrm{Z}) \mathrm{QX} \\
& +\mathrm{S}(\phi \mathrm{X}, \phi \mathrm{Z}) \phi \mathrm{Y}-\mathrm{S}(\phi \mathrm{Y}, \phi \mathrm{Z}) \phi \mathrm{X}+g(\phi \mathrm{X}, \phi \mathrm{Z}) \mathrm{Q} \phi \mathrm{Y}-g(\phi \mathrm{Y}, \phi \mathrm{Z}) \mathrm{Q} \phi \mathrm{X}-2 \mathrm{~S}(\phi \mathrm{X}, \mathrm{Y}) \mathrm{Z} \\
& +2 \mathrm{~S}(\phi \mathrm{X}, \mathrm{Y}) \eta(\mathrm{Z}) \xi-2 g(\phi \mathrm{X}, \mathrm{Y}) \mathrm{QZ}+2 g(\mathrm{X}, \mathrm{Y}) \eta(\mathrm{Z}) \mathrm{Q} \xi-\mathrm{S}(\mathrm{X}, \phi \mathrm{Z}) \eta(\mathrm{Y}) \xi+\mathrm{S}(\mathrm{Y}, \phi \mathrm{Z}) \eta(\mathrm{X}) \xi \\
& -\mathrm{S}(\mathrm{X}, \mathrm{Z}) \phi \mathrm{Y}+\mathrm{S}(\mathrm{Y}, \mathrm{Z}) \phi \mathrm{X}-g(\mathrm{X}, \mathrm{Z}) \mathrm{Q} \phi \mathrm{Y}+g(\mathrm{Y}, \mathrm{Z}) \mathrm{Q} \phi \mathrm{X}+\mathrm{S}(\phi \mathrm{X}, \mathrm{Z}) \mathrm{Y}-\mathrm{S}(\phi \mathrm{X}, \mathrm{Z}) \eta(\mathrm{Y}) \xi \\
& -\mathrm{S}(\phi \mathrm{Y}, \mathrm{Z}) \mathrm{X}+\mathrm{S}(\phi \mathrm{Y}, \mathrm{Z}) \eta(\mathrm{X}) \xi+g(\phi \mathrm{X}, \mathrm{Z}) \mathrm{QY}-g(\phi \mathrm{X}, \mathrm{Z}) \eta(\mathrm{Y}) \mathrm{Q} \xi-g(\phi \mathrm{Y}, \mathrm{Z}) \mathrm{QX} \\
& +g(\phi \mathrm{Y}, \mathrm{Z}) \eta(\mathrm{X}) \mathrm{Q} \xi+2 \mathrm{~S}(\phi \mathrm{X}, \mathrm{Y}) \mathrm{Z}-2 \mathrm{~S}(\phi \mathrm{X}, \mathrm{Y}) \eta(\mathrm{Z}) \xi-2 g(\phi \mathrm{X}, \mathrm{Y}) \mathrm{QZ}+2 g(\phi \mathrm{X}, \mathrm{Y}) \eta(\mathrm{Z}) \mathrm{Q} \xi \\
& -\eta(\mathrm{X}) \eta(\mathrm{Z}) \mathrm{Q} \phi \mathrm{Y}+\eta(\mathrm{Y}) \eta(\mathrm{Z}) \mathrm{Q} \phi \mathrm{X}]-\frac{\mathrm{p}+2 \mathrm{n}}{2 \mathrm{n}+4}[g(\mathrm{X}, \mathrm{Z}) \phi \mathrm{Y}-\eta(\mathrm{X}) \eta(\mathrm{Z}) \phi \mathrm{Y}-g(\mathrm{Y}, \mathrm{Z}) \phi \mathrm{X} \\
& +\eta(\mathrm{Y}) \eta(\mathrm{Z}) \phi \mathrm{X}-2 g(\phi \mathrm{X}, \mathrm{Y}) \mathrm{Z}+2 g(\mathrm{X}, \mathrm{Y}) \eta(\mathrm{Z}) \xi+g(\phi \mathrm{X}, \mathrm{Z}) \mathrm{Y}-g(\phi \mathrm{X}, \mathrm{Z}) \eta(\mathrm{Y}) \xi \\
& -g(\phi \mathrm{Y}, \mathrm{Z}) \mathrm{X}+g(\phi \mathrm{Y}, \mathrm{Z}) \eta(\mathrm{X}) \xi+2 g(\phi \mathrm{X}, \mathrm{Y}) \mathrm{Z}-2 g(\phi \mathrm{X}, \mathrm{Y}) \eta(\mathrm{Z}) \xi]-\frac{\mathrm{p}-4}{2 \mathrm{n}+4}[g(\mathrm{X}, \phi \mathrm{Z}) \mathrm{Y} \\
& -g(\mathrm{Y}, \phi \mathrm{Z}) \mathrm{X}-g(\mathrm{X}, \mathrm{Z}) \phi \mathrm{Y}+g(\mathrm{Y}, \mathrm{Z}) \phi \mathrm{X}]+\frac{\mathrm{p}}{2 \mathrm{n}+4}[g(\mathrm{X}, \phi \mathrm{Z}) \eta(\mathrm{Y}) \xi+g(\mathrm{Y}, \phi \mathrm{Z}) \eta(\mathrm{X}) \xi \\
& -\eta(\mathrm{X}) \eta(\mathrm{Z}) \phi \mathrm{Y}-\eta(\mathrm{Y}) \eta(\mathrm{Z}) \phi \mathrm{X}]=0 .
\end{aligned}
$$

Now replacing $X$ by $\phi X$ in (4.3) and making use of (2.1), (2.4) and (2.11), we have

$$
\begin{aligned}
& (1-\kappa) g(\mathrm{X}, \mathrm{Z}) \eta(\mathrm{Y}) \xi-(1-\kappa) \eta(\mathrm{X}) \eta(\mathrm{Y}) \eta(\mathrm{Z}) \xi-(1-\mu) g(h \mathrm{X}, \mathrm{Z}) \eta(\mathrm{Y}) \xi+g(\mathrm{Y}, \mathrm{Z}) \mathrm{X} \\
& -g(\mathrm{Y}, \mathrm{Z}) \eta(\mathrm{X}) \xi-g(\mathrm{Y}, \mathrm{Z}) h \mathrm{X}+g(h \mathrm{Y}, \mathrm{Z}) \mathrm{X}-g(h \mathrm{Y}, \mathrm{Z}) \eta(\mathrm{X}) \xi-g(h \mathrm{Y}, \mathrm{Z}) h \mathrm{X} \\
& +g(\phi \mathrm{X}, \mathrm{Z}) \phi \mathrm{Y}+g(\phi \mathrm{X}, \mathrm{Z}) \phi h \mathrm{Y}+g(h \phi \mathrm{X}, \mathrm{Z}) \phi \mathrm{Y}+g(h \phi \mathrm{X}, \mathrm{Z}) \phi h \mathrm{Y}-g(\phi \mathrm{Y}, \mathrm{Z}) \phi \mathrm{X} \\
& -g(\phi \mathrm{Y}, \mathrm{Z}) h \phi \mathrm{X}-g(\phi h \mathrm{Y}, \mathrm{Z}) \phi \mathrm{X}-g(\phi h \mathrm{Y}, \mathrm{Z}) h \phi \mathrm{X}-g(\mathrm{X}, \mathrm{Z}) \mathrm{Y}+\eta(\mathrm{X}) \eta(\mathrm{Z}) \mathrm{Y} \\
& +g(h \mathrm{X}, \mathrm{Z}) \mathrm{Y}-g(\mathrm{X}, \mathrm{Z}) h \mathrm{Y}+\eta(\mathrm{X}) \eta(\mathrm{Z}) h \mathrm{Y}+g(h \mathrm{X}, \mathrm{Z}) h \mathrm{Y}-(1-\kappa) \eta(\mathrm{Y}) \eta(\mathrm{Z}) \mathrm{X} \\
& +(1-\kappa) \eta(\mathrm{X}) \eta(\mathrm{Y}) \eta(\mathrm{Z}) \xi+(1-\mu) \eta(\mathrm{Y}) \eta(\mathrm{Z}) h \mathrm{X}+\frac{1}{2 \mathrm{n}+4}[\mathrm{~S}(\mathrm{X}, \mathrm{Z}) \mathrm{Y}-2 \mathrm{n} \kappa \eta(\mathrm{X}) \eta(\mathrm{Z}) \mathrm{Y} \\
& -2(2 \mathrm{n}-2+\mu) g(h \mathrm{X}, \mathrm{Z}) \mathrm{Y}-\mathrm{S}(\mathrm{Y}, \phi \mathrm{Z}) \phi \mathrm{X}+g(\mathrm{X}, \mathrm{Z}) \mathrm{QY}-\eta(\mathrm{X}) \eta(\mathrm{Z}) \mathrm{QY}-g(\mathrm{Y}, \phi \mathrm{Z}) \mathrm{Q} \phi \mathrm{X}
\end{aligned}
$$




$$
\begin{aligned}
& -\mathrm{S}(\mathrm{X}, \phi \mathrm{Z}) \phi \mathrm{Y}+\mathrm{S}(\mathrm{Y}, \mathrm{Z}) \mathrm{X}-2 \mathrm{n} \kappa \eta(\mathrm{Y}) \eta(\mathrm{Z}) \mathrm{X}-2(2 \mathrm{n}-2+\mu) g(h \mathrm{Y}, \mathrm{Z}) \mathrm{X}-\mathrm{S}(\mathrm{Y}, \mathrm{Z}) \eta(\mathrm{X}) \xi \\
& +2 \mathrm{n} \kappa(\mathrm{X}) \eta(\mathrm{Y}) \eta(\mathrm{Z}) \xi+2(2 \mathrm{n}-2+\mu) g(h \mathrm{Y}, \mathrm{Z}) \eta(\mathrm{X}) \xi-g(\mathrm{X}, \phi \mathrm{Z}) \mathrm{Q} \phi \mathrm{Y}+\mathrm{g}(\mathrm{Y}, \mathrm{Z}) \mathrm{QX} \\
& -g(\mathrm{Y}, \mathrm{Z}) \eta(\mathrm{X}) \mathrm{Q} \xi-\eta(\mathrm{Y}) \eta(\mathrm{Z}) \mathrm{QX}+\eta(\mathrm{X}) \eta(\mathrm{Y}) \eta(\mathrm{Z}) \xi+2 \mathrm{~S}(\mathrm{X}, \mathrm{Y}) \mathrm{Z}-4 \mathrm{n} \kappa \eta(\mathrm{X}) \eta(\mathrm{Y}) \mathrm{Z} \\
& -2 \mathrm{~S}(\mathrm{X}, \mathrm{Y}) \eta(\mathrm{Z}) \xi+4 \mathrm{n} \kappa \eta(\mathrm{X}) \eta(\mathrm{Y}) \eta(\mathrm{Z}) \xi+2 g(\mathrm{X}, \mathrm{Y}) \mathrm{QZ}+2 \eta(\mathrm{X}) \eta(\mathrm{Y}) \mathrm{QZ}-2 g(\mathrm{X}, \mathrm{Y}) \eta(\mathrm{Z}) \xi \\
& +2 \eta(\mathrm{X}) \eta(\mathrm{Y}) \eta(\mathrm{Z}) \xi-\mathrm{S}(\mathrm{X}, \mathrm{Z}) \eta(\mathrm{Y}) \xi+2 \mathrm{n} \kappa \eta(\mathrm{X}) \eta(\mathrm{Y}) \eta(\mathrm{Z}) \xi+2(2 \mathrm{n}-2+\mu) g(\mathrm{hX}, \mathrm{Z}) \eta(\mathrm{Y}) \xi \\
& -\mathrm{S}(\phi \mathrm{X}, \mathrm{Z}) \phi \mathrm{Y}-\mathrm{S}(\mathrm{Y}, \mathrm{Z}) \mathrm{X}+\mathrm{S}(\mathrm{Y}, \mathrm{Z}) \eta(\mathrm{X}) \xi-g(\phi \mathrm{X}, \mathrm{Z}) \mathrm{Q} \phi \mathrm{Y}-g(\mathrm{Y}, \mathrm{Z}) \mathrm{QX}+g(\mathrm{Y}, \mathrm{Z}) \eta(\mathrm{X}) \mathrm{Q} \xi \\
& -\mathrm{S}(\mathrm{X}, \mathrm{Z}) \mathrm{Y}-2 \mathrm{n} \kappa \eta(\mathrm{X}) \eta(\mathrm{Z}) \mathrm{Y}-\mathrm{S}(\mathrm{X}, \mathrm{Z}) \eta(\mathrm{Y}) \xi+2 \mathrm{n} \kappa \eta(\mathrm{X}) \eta(\mathrm{Y}) \eta(\mathrm{Z}) \xi-\mathrm{S}(\phi \mathrm{Y}, \mathrm{Z}) \phi \mathrm{X}-g(\mathrm{X}, \mathrm{Z}) \mathrm{QY} \\
& +\eta(\mathrm{X}) \eta(\mathrm{Z}) \mathrm{QY}+g(\mathrm{X}, \mathrm{Z}) \eta(\mathrm{Y}) \mathrm{Q} \xi-\eta(\mathrm{X}) \eta(\mathrm{Y}) \eta(\mathrm{Z}) \mathrm{Q} \xi-g(\mathrm{Y}, \mathrm{Z}) \mathrm{Q} \phi \mathrm{X}-2 \mathrm{~S}(\mathrm{X}, \mathrm{Y}) \mathrm{Z} \\
& +4 \mathrm{n} \kappa \eta(\mathrm{X}) \eta(\mathrm{Y}) \mathrm{Z}+2 \mathrm{~S}(\mathrm{X}, \mathrm{Y}) \eta(\mathrm{Z}) \xi-4 \mathrm{n} \eta(\mathrm{X}) \eta(\mathrm{Y}) \eta(\mathrm{Z}) \xi+2 g(\mathrm{X}, \mathrm{Y}) \mathrm{QZ}-2 \eta(\mathrm{X}) \eta(\mathrm{Y}) \mathrm{QZ} \\
& -2 g(\mathrm{X}, \mathrm{Y}) \eta(\mathrm{Z}) \mathrm{Q} \xi+2 \eta(\mathrm{X}) \eta(\mathrm{Y}) \eta(\mathrm{Z}) \mathrm{Q} \xi-\eta(\mathrm{Y}) \eta(\mathrm{Z}) \mathrm{QX}+\eta(\mathrm{X}) \eta(\mathrm{Y}) \eta(\mathrm{Z}) \xi] \\
& -\frac{\mathrm{p}+2 \mathrm{n}}{2 \mathrm{n}+4}[g(\phi \mathrm{X}, \mathrm{Z}) \phi \mathrm{Y}+g(\mathrm{Y}, \mathrm{Z}) \mathrm{X}-g(\mathrm{Y}, \mathrm{Z}) \eta(\mathrm{X}) \xi-\eta(\mathrm{Y}) \eta(\mathrm{Z}) \mathrm{X}+\eta(\mathrm{X}) \eta(\mathrm{Y}) \eta(\mathrm{Z}) \xi+2 g(\mathrm{X}, \mathrm{Y}) \mathrm{Z} \\
& -2 \eta(\mathrm{X}) \eta(\mathrm{Y}) \mathrm{Z}-2 g(\mathrm{X}, \mathrm{Y}) \eta(\mathrm{Z}) \xi+2 \eta(\mathrm{X}) \eta(\mathrm{Y}) \eta(\mathrm{Z}) \xi-g(\mathrm{X}, \mathrm{Z}) \mathrm{Y}+\eta(\mathrm{X}) \eta(\mathrm{Z}) \mathrm{Y}+g(\mathrm{X}, \mathrm{Z})(\mathrm{Y}) \xi \\
& -\eta(\mathrm{X}) \eta(\mathrm{Y}) \eta(\mathrm{Z}) \xi-g(\phi \mathrm{Y}, \mathrm{Z}) \phi \mathrm{X}-2 g(\mathrm{X}, \mathrm{Y}) \mathrm{Z}+2 \eta(\mathrm{X}) \eta(\mathrm{Y}) \mathrm{Z}+2 g(\mathrm{X}, \mathrm{Y}) \eta(\mathrm{Z}) \xi \\
& -2 \eta(\mathrm{X}) \eta(\mathrm{Y}) \eta(\mathrm{Z}) \xi]-\frac{\mathrm{p}-4}{2 \mathrm{n}+4}[g(\mathrm{X}, \mathrm{Z}) \mathrm{Y}-\eta(\mathrm{X}) \eta(\mathrm{Z}) \mathrm{Y}-g(\mathrm{Y}, \phi \mathrm{Z}) \phi \mathrm{X}-g(\phi \mathrm{X}, \mathrm{Z}) \phi \mathrm{Y} \\
& -g(\mathrm{Y}, \mathrm{Z}) \mathrm{X}+g(\mathrm{Y}, \mathrm{Z}) \eta(\mathrm{X}) \xi]+\frac{\mathrm{p}}{2 \mathrm{n}+4}[g(\mathrm{X}, \mathrm{Z}) \eta(\mathrm{Y}) \xi-\eta(\mathrm{Y}) \eta(\mathrm{Z}) \mathrm{X}]=0 .
\end{aligned}
$$

By taking inner product of (4.4) with $T$ and using symmetry of $h,(2.1),(2.9)$ and (2.12), we obtain

$$
\begin{aligned}
& (1-\kappa) g(\mathrm{X}, \mathrm{Z}) \eta(\mathrm{Y}) \eta(\mathrm{T})-(1-\kappa) \eta(\mathrm{X}) \eta(\mathrm{Y}) \eta(\mathrm{Z}) \eta(\mathrm{T})-(1-\mu) g(h \mathrm{X}, \mathrm{Z}) \eta(\mathrm{Y}) \eta(\mathrm{T}) \\
& +g(\mathrm{Y}, \mathrm{Z}) g(\mathrm{X}, \mathrm{T})-g(\mathrm{Y}, \mathrm{Z}) \eta(\mathrm{X}) \eta(\mathrm{T})-g(\mathrm{Y}, \mathrm{Z}) g(h \mathrm{X}, \mathrm{T})+g(h \mathrm{Y}, \mathrm{Z}) g(\mathrm{X}, \mathrm{T}) \\
& -g(h \mathrm{Y}, \mathrm{Z}) \eta(\mathrm{X}) \eta(\mathrm{T})-g(h \mathrm{Y}, \mathrm{Z}) g(h \mathrm{X}, \mathrm{T})+g(\phi \mathrm{X}, \mathrm{Z}) g(\phi \mathrm{Y}, \mathrm{T})+g(\phi \mathrm{X}, \mathrm{Z}) g(\phi h \mathrm{Y}, \mathrm{T}) \\
& +g(\phi h \mathrm{X}, \mathrm{Z}) g(\phi \mathrm{Y}, \mathrm{T})+g(h \phi \mathrm{X}, \mathrm{Z}) g(\phi h \mathrm{Y}, \mathrm{T})-g(\phi \mathrm{Y}, \mathrm{Z}) g(\phi \mathrm{X}, \mathrm{T})-g(\phi \mathrm{Y}, \mathrm{Z}) g(h \phi \mathrm{X}, \mathrm{T}) \\
& -g(\phi h \mathrm{Y}, \mathrm{Z}) g(\phi \mathrm{X}, \mathrm{T})-g(\phi h \mathrm{Y}, \mathrm{Z}) g(h \phi \mathrm{X}, \mathrm{T})-g(\mathrm{X}, \mathrm{Z}) g(\mathrm{Y}, \mathrm{T})+\eta(\mathrm{X}) \eta(\mathrm{Z}) g(\mathrm{Y}, \mathrm{T}) \\
& +g(h \mathrm{X}, \mathrm{Z}) g(\mathrm{Y}, \mathrm{T})-g(\mathrm{X}, \mathrm{Z}) g(h \mathrm{Y}, \mathrm{T})+\eta(\mathrm{X}) \eta(\mathrm{Z}) g(h \mathrm{Y}, \mathrm{T})+g(h \mathrm{X}, \mathrm{Z}) g(h \mathrm{Y}, \mathrm{T}) \\
& -(1-\kappa) \eta(\mathrm{Y}) \eta(\mathrm{Z}) g(\mathrm{X}, \mathrm{T})+(1-\kappa) \eta(\mathrm{X}) \eta(\mathrm{Y}) \eta(\mathrm{Z}) \eta(\mathrm{T})+(1-\mu) \eta(\mathrm{Y}) \eta(\mathrm{Z}) g(h \mathrm{X}, \mathrm{T}) \\
& +\frac{1}{2 \mathrm{n}+4}[\mathrm{~S}(\mathrm{X}, \mathrm{Z}) g(\mathrm{Y}, \mathrm{T})-2 \mathrm{n} \kappa \eta(\mathrm{X}) \eta(\mathrm{Z}) g(\mathrm{Y}, \mathrm{T})-2(2 \mathrm{n}-2+\mu) g(h \mathrm{X}, \mathrm{Z}) g(\mathrm{Y}, \mathrm{T}) \\
& -\mathrm{S}(\mathrm{Y}, \phi \mathrm{Z}) g(\phi \mathrm{X}, \mathrm{T})+g(\mathrm{X}, \mathrm{Z}) \mathrm{S}(\mathrm{Y}, \mathrm{T})-\eta(\mathrm{X}) \eta(\mathrm{Z}) \mathrm{S}(\mathrm{Y}, \mathrm{T})-g(\mathrm{Y}, \phi \mathrm{Z}) \mathrm{S}(\phi \mathrm{X}, \mathrm{T}) \\
& -\mathrm{S}(\mathrm{X}, \phi \mathrm{Z}) g(\phi \mathrm{Y}, \mathrm{T})+\mathrm{S}(\mathrm{Y}, \mathrm{Z}) g(\mathrm{X}, \mathrm{T})-2 \mathrm{n} \kappa \eta(\mathrm{Y}) \eta(\mathrm{Z}) g(\mathrm{X}, \mathrm{T})-2(2 \mathrm{n}-2+\mu)
\end{aligned}
$$




$$
\begin{aligned}
& g(h \mathrm{Y}, \mathrm{Z}) g(\mathrm{X}, \mathrm{T})-\mathrm{S}(\mathrm{Y}, \mathrm{Z}) \eta(\mathrm{X}) \eta(\mathrm{T})+2 \mathrm{n} \kappa \eta(\mathrm{X}) \eta(\mathrm{Y}) \eta(\mathrm{Z}) \eta(\mathrm{T})+2(2 \mathrm{n}-2+\mu) \\
& g(\mathrm{hY}, \mathrm{Z}) \eta(\mathrm{X}) \eta(\mathrm{T})-g(\mathrm{X}, \phi \mathrm{Z}) \mathrm{S}(\phi \mathrm{Y}, \mathrm{T})+g(\mathrm{Y}, \mathrm{Z}) \mathrm{S}(\mathrm{X}, \mathrm{T})-2 \mathrm{n} \kappa g(\mathrm{Y}, \mathrm{Z}) \eta(\mathrm{X}) \eta(\mathrm{T}) \\
& -2 \mathrm{n} \kappa \eta(\mathrm{Y}) \eta(\mathrm{Z}) g(\mathrm{X}, \mathrm{T})+\eta(\mathrm{X}) \eta(\mathrm{Y}) \eta(\mathrm{Z}) \eta(\mathrm{T})+2 \mathrm{~S}(\mathrm{X}, \mathrm{Y}) g(\mathrm{Z}, \mathrm{T})-4 \mathrm{n} \kappa \eta(\mathrm{X}) \eta(\mathrm{Y}) g(\mathrm{Z}, \mathrm{T}) \\
& -2 \mathrm{~S}(\mathrm{X}, \mathrm{Y}) \eta(\mathrm{Z}) \eta(\mathrm{T})+4 \mathrm{n} \kappa \eta(\mathrm{X}) \eta(\mathrm{Y}) \eta(\mathrm{Z}) \eta(\mathrm{T})+2 g(\mathrm{X}, \mathrm{Y}) \mathrm{S}(\mathrm{Z}, \mathrm{T})+2 \eta(\mathrm{X}) \eta(\mathrm{Y}) \mathrm{S}(\mathrm{Z}, \mathrm{T}) \\
& -2 g(\mathrm{X}, \mathrm{Y}) \eta(\mathrm{Z}) \eta(\mathrm{T})+2 \eta(\mathrm{X}) \eta(\mathrm{Y}) \eta(\mathrm{Z}) \eta(\mathrm{T})-\mathrm{S}(\mathrm{X}, \mathrm{Z}) \eta(\mathrm{Y}) \eta(\mathrm{T})+2 \mathrm{n} \kappa \eta(\mathrm{X}) \eta(\mathrm{Y}) \eta(\mathrm{Z}) \eta(\mathrm{T}) \\
& +2(2 \mathrm{n}-2+\mu) g(h \mathrm{X}, \mathrm{Z}) \eta(\mathrm{Y}) \eta(\mathrm{T})-\mathrm{S}(\phi \mathrm{X}, \mathrm{Z}) g(\phi \mathrm{Y}, \mathrm{T})-\mathrm{S}(\mathrm{Y}, \mathrm{Z}) g(\mathrm{X}, \mathrm{T})+\mathrm{S}(\mathrm{Y}, \mathrm{Z}) \eta(\mathrm{X}) \eta(\mathrm{T}) \\
& -g(\phi \mathrm{X}, \mathrm{Z}) \mathrm{S}(\phi \mathrm{Y}, \mathrm{T})-g(\mathrm{Y}, \mathrm{Z}) \mathrm{S}(\mathrm{X}, \mathrm{T})+2 \mathrm{n} \kappa g(\mathrm{Y}, \mathrm{Z}) \eta(\mathrm{X}) \eta(\mathrm{T})-\mathrm{S}(\mathrm{X}, \mathrm{Z}) g(\mathrm{Y}, \mathrm{T}) \\
& -2 \mathrm{n} \kappa \eta(\mathrm{X}) \eta(\mathrm{Z}) g(\mathrm{Y}, \mathrm{T})+\mathrm{S}(\mathrm{X}, \mathrm{Z}) \eta(\mathrm{Y}) \eta(\mathrm{T})+2 \mathrm{n} \kappa \eta(\mathrm{X}) \eta(\mathrm{Y}) \eta(\mathrm{Z}) \eta(\mathrm{T})-\mathrm{S}(\phi \mathrm{Y}, \mathrm{Z}) g(\phi \mathrm{X}, \mathrm{T}) \\
& -g(\mathrm{X}, \mathrm{Z}) \mathrm{S}(\mathrm{Y}, \mathrm{T})+\eta(\mathrm{X}) \eta(\mathrm{Z}) \mathrm{S}(\mathrm{Y}, \mathrm{T})+2 \mathrm{n} \kappa g(\mathrm{X}, \mathrm{Z}) \eta(\mathrm{Y}) \eta(\mathrm{T})-\eta(\mathrm{X}) \eta(\mathrm{Y}) \eta(\mathrm{Z}) \eta(\mathrm{T}) \\
& -g(\phi \mathrm{Y}, \mathrm{Z}) \mathrm{S}(\phi \mathrm{Y}, \mathrm{T})-2 \mathrm{~S}(\mathrm{X}, \mathrm{Y}) g(\mathrm{Z}, \mathrm{T})-4 \mathrm{n} \kappa \eta(\mathrm{X}) \eta(\mathrm{Y}) g(\mathrm{Z}, \mathrm{T})+2 \mathrm{~S}(\mathrm{X}, \mathrm{Y}) \eta(\mathrm{Z}) \eta(\mathrm{T}) \\
& -4 \mathrm{n} \kappa(\mathrm{X}) \eta(\mathrm{Y}) \eta(\mathrm{Z}) \eta(\mathrm{T})+2 g(\mathrm{X}, \mathrm{Y}) \mathrm{S}(\mathrm{Z}, \mathrm{T})-2 \eta(\mathrm{X}) \eta(\mathrm{Y}) \mathrm{S}(\mathrm{Z}, \mathrm{T})-4 \mathrm{n} \kappa g(\mathrm{X}, \mathrm{Y}) \eta(\mathrm{Z}) \eta(\mathrm{T}) \\
& +4 \mathrm{n} \kappa \eta(\mathrm{X}) \eta(\mathrm{Y}) \eta(\mathrm{Z}) \eta(\mathrm{T})-2 \mathrm{n} \kappa \eta(\mathrm{Y}) \eta(\mathrm{Z}) g(\mathrm{X}, \mathrm{T})+\eta(\mathrm{X}) \eta(\mathrm{Y}) \eta(\mathrm{Z}) \eta(\mathrm{T})] \\
& -\frac{\mathrm{p}+2 \mathrm{n}}{2 \mathrm{n}+4}[g(\phi \mathrm{X}, \mathrm{Z}) g(\phi \mathrm{Y}, \mathrm{T})+g(\mathrm{Y}, \mathrm{Z}) g(\mathrm{X}, \mathrm{T})-g(\mathrm{Y}, \mathrm{Z}) \eta(\mathrm{X}) \eta(\mathrm{T})-\eta(\mathrm{Y}) \eta(\mathrm{Z}) g(\mathrm{X}, \mathrm{T}) \\
& +\eta(\mathrm{X}) \eta(\mathrm{Y}) \eta(\mathrm{Z}) \eta(\mathrm{T})+2 g(\mathrm{X}, \mathrm{Y}) g(\mathrm{Z}, \mathrm{T})-2 \eta(\mathrm{X}) \eta(\mathrm{Y}) g(\mathrm{Z}, \mathrm{T})-2 g(\mathrm{X}, \mathrm{Y}) \eta(\mathrm{Z}) \eta(\mathrm{T}) \\
& +2 \eta(\mathrm{X}) \eta(\mathrm{Y}) \eta(\mathrm{Z}) \eta(\mathrm{T})-g(\mathrm{X}, \mathrm{Z}) g(\mathrm{Y}, \mathrm{T})+\eta(\mathrm{X}) \eta(\mathrm{Z}) g(\mathrm{Z}, \mathrm{T})+g(\mathrm{X}, \mathrm{Z}) \eta(\mathrm{Y}) \eta(\mathrm{T}) \\
& -\eta(\mathrm{X}) \eta(\mathrm{Y}) \eta(\mathrm{Z})(\mathrm{T})-g(\phi \mathrm{Y}, \mathrm{Z}) g(\phi \mathrm{X}, \mathrm{T})-2 g(\mathrm{X}, \mathrm{Y}) g(\mathrm{Z}, \mathrm{T})+2 \eta(\mathrm{X}) \eta(\mathrm{Y}) g(\mathrm{Z}, \mathrm{T}) \\
& +2 g(\mathrm{X}, \mathrm{Y}) \eta(\mathrm{Z}) \eta(\mathrm{T})-2 \eta(\mathrm{X}) \eta(\mathrm{Y}) \eta(\mathrm{Z}) \eta(\mathrm{T})]-\frac{\mathrm{p}-4}{2 \mathrm{n}+4}[g(\mathrm{X}, \mathrm{Z}) g(\mathrm{Y}, \mathrm{T}) \\
& -\eta(\mathrm{X}) \eta(\mathrm{Z}) g(\mathrm{Y}, \mathrm{T})-g(\mathrm{Y}, \phi \mathrm{Z}) g(\phi \mathrm{X}, \mathrm{T})-g(\phi \mathrm{X}, \mathrm{Z}) g(\phi \mathrm{Y}, \mathrm{T})-g(\mathrm{Y}, \mathrm{Z}) g(\mathrm{X}, \mathrm{T}) \\
& +g(\mathrm{Y}, \mathrm{Z}) \eta(\mathrm{X}) \eta(\mathrm{T})]+\frac{\mathrm{p}}{2 \mathrm{n}+4}[g(\mathrm{X}, \mathrm{Z}) \eta(\mathrm{Y}) \eta(\mathrm{T})-\eta(\mathrm{Y}) \eta(\mathrm{Z}) g(\mathrm{X}, \mathrm{T})]=0 .
\end{aligned}
$$

Now plugging $Y=T=\xi$ in (4.5) and then using equations (2.4) and (2.5), we get

$$
\frac{4-4 \kappa}{2 n+4} g(X, Z)+\frac{\kappa(6 n+4)}{2 n+4} \eta(X) \eta(Z)+\frac{\mu(2 n+4)-2(2 n-2+\mu)}{2 n+4} g(h X, Z)=0 .
$$

Further making use of (2.10), (4.6) leads to

$$
S(X, Z)=A_{3} g(X, Z)+A_{4} \eta(X) \eta(Z),
$$

where

$A_{3}=\frac{[2(n-1)-n \mu]\{\mu(2 n+4)-2(2(n-1)+\mu)\}+4(\kappa-1)(2 n+4)[2(n-1)+\mu]}{\mu(2 n+4)-2(2 n-2+\mu)}$ and 


$$
A_{4}=\frac{[2(1-n)+n(2 \kappa+\mu)]\{\mu(2 n+4)-2(2(n-1)+\mu)\}-\{\kappa(6 n+4)-3\}(2 n+4)[2(n-1)+\mu]}{\mu(2 n+4)-2(2(n-1)+\mu)}
$$

Hence we can state the following:

Theorem 4.3. If $(2 n+1)$-dimensional non-Sasakian $(\kappa, \mu)$-contact metric manifold $M$ is $\phi$ - $C$-Bochner semisymmetric then $M^{2 n+1}$ is an $\eta$-Einstein manifold with constant coefficients.

Similarly, from proposition 2.1 and Theorem 4.3 we can state the following:

Corollary 4.3. If $\mathrm{M}$ be a $\phi$-C-Bochner semisymmetric $(\kappa, \mu)$-contact metric manifold then the Ricci operator $Q$ commutes with $\phi$ i.e., $Q \phi=\phi Q$.

\section{5. $\boldsymbol{h}$-m-projectively semisymmetric non-Sasakian $(\kappa, \mu)$-contact metric manifold}

Definition 5.3. A Riemannian manifold $\left(M^{2 n+1}, g\right)$ is said to be $h$-m-projectively semisymmetric if the condition,

$$
\mathrm{W}^{*}(\mathrm{X}, \mathrm{Y}) \cdot h=0,
$$

holds on $M$.

Let $M$ be a $(2 n+1)$-dimensional $h$-m-projectively semisymmetric non-Sasakian $(\kappa, \mu)$-contact metric manifold. The condition $\mathrm{W}^{*}(\mathrm{X}, \mathrm{Y}) \cdot h=0$ can be written as,

$$
\left(\mathrm{W}^{*}(\mathrm{X}, \mathrm{Y}) \cdot h\right) \mathrm{Z}=\mathrm{W}^{*}(\mathrm{X}, \mathrm{Y}) h \mathrm{Z}-h \mathrm{~W}^{*}(\mathrm{X}, \mathrm{Y}) \mathrm{Z}=0,
$$

for any vector fields $X, Y, Z$.

By using (1.2) and (2.14) in (5.2), we get

$$
\begin{aligned}
& \{\kappa[g(h \mathrm{Y}, \mathrm{Z}) \eta(\mathrm{X})-g(h \mathrm{X}, \mathrm{Z}) \eta(\mathrm{Y})]+\mu(\kappa-1)[g(\mathrm{X}, \mathrm{Z}) \eta(\mathrm{Y})-g(\mathrm{Y}, \mathrm{Z}) \eta(\mathrm{X})]\} \xi \\
& +\kappa\{g(\mathrm{Y}, \phi \mathrm{Z}) \phi h \mathrm{X}-g(\mathrm{X}, \phi \mathrm{Z}) \phi h \mathrm{Y}+g(\mathrm{Z}, \phi h \mathrm{Y}) \phi \mathrm{X}-g(\mathrm{Z}, \phi h \mathrm{X}) \phi \mathrm{Y}+\eta(\mathrm{Z})[\eta(\mathrm{X}) h \mathrm{Y} \\
& -\eta(\mathrm{Y}) h \mathrm{X}]\}-\mu\{\eta(\mathrm{Y})[(1-\kappa) \eta(\mathrm{Z}) \mathrm{X}+\mu \eta(\mathrm{X}) h \mathrm{Z}]-\eta(\mathrm{X})[(1-\kappa) \eta(\mathrm{Z}) \mathrm{Y}+\mu \eta(\mathrm{Y}) h \mathrm{Z}] \\
& +2 g(\mathrm{X}, \phi \mathrm{Y}) \phi h \mathrm{Z}\}-\frac{1}{4 \mathrm{n}}[\mathrm{S}(\mathrm{Y}, h \mathrm{Z}) \mathrm{X}-\mathrm{S}(\mathrm{X}, h \mathrm{Z}) \mathrm{Y}+g(\mathrm{Y}, h \mathrm{Z}) \mathrm{QX}-g(\mathrm{X}, h \mathrm{Z}) \mathrm{QY} \\
& -\mathrm{S}(\mathrm{Y}, \mathrm{Z}) h \mathrm{X}+\mathrm{S}(\mathrm{X}, \mathrm{Z}) h \mathrm{Y}-g(\mathrm{Y}, \mathrm{Z}) h \mathrm{QX}+g(\mathrm{X}, \mathrm{Z}) h \mathrm{QY}]=0 .
\end{aligned}
$$

Replacing $X$ by $h X$ in (5.3) and using symmetric property of $h,(2.1),(2.7)$, we obtain

$$
\begin{aligned}
& \kappa(\kappa-1) g(\mathrm{X}, \mathrm{Z}) \eta(\mathrm{Y}) \xi-\kappa(\kappa-1) \eta(\mathrm{X}) \eta(\mathrm{Y}) \eta(\mathrm{Z}) \xi+\mu(\kappa-1) g(h \mathrm{X}, \mathrm{Z}) \eta(\mathrm{Y}) \xi \\
& -\kappa(\kappa-1) g(\mathrm{Y}, \phi \mathrm{Z}) \phi \mathrm{X}-\kappa g(h \mathrm{X}, \phi \mathrm{Z}) \phi h \mathrm{Y}+\kappa g(\mathrm{Z}, \phi h \mathrm{Y}) \phi h \mathrm{X}+\kappa(\kappa-1) g(\mathrm{Z}, \phi \mathrm{X}) \phi \mathrm{Y} \\
& +\kappa(\kappa-1) \eta(\mathrm{Y}) \eta(\mathrm{Z}) \mathrm{X}-\kappa(\kappa-1) \eta(\mathrm{X}) \eta(\mathrm{Y}) \eta(\mathrm{Z}) \xi-\mu(1-\kappa) \eta(\mathrm{Y}) \eta(\mathrm{Z}) h \mathrm{X}-2 \mu g(h \mathrm{X}, \phi \mathrm{Y}) \phi h \mathrm{Z} \\
& -\frac{1}{4 \mathrm{n}}[\mathrm{S}(\mathrm{Y}, h \mathrm{Z}) h \mathrm{X}-\mathrm{S}(h \mathrm{X}, h \mathrm{Z}) \mathrm{Y}+g(\mathrm{Y}, h \mathrm{Z}) \mathrm{Q} h \mathrm{X}-g(h \mathrm{X}, h \mathrm{Z}) \mathrm{QY}+(\kappa-1) \mathrm{S}(\mathrm{Y}, \mathrm{Z}) \mathrm{X} \\
& -(\kappa-1) \mathrm{S}(\mathrm{Y}, \mathrm{Z}) \eta(\mathrm{X}) \xi+\mathrm{S}(h \mathrm{X}, \mathrm{Z}) h \mathrm{Y}+(\kappa-1) g(\mathrm{Y}, \mathrm{Z}) \mathrm{QX}-(\kappa-1) g(\mathrm{Y}, \mathrm{Z}) \eta(\mathrm{X}) \mathrm{Q} \xi \\
& +g(h X, Z) Q h Y]=0 .
\end{aligned}
$$

Taking inner product of (5.4) with $T$ and then using equations (2.1) and (2.12), symmetry of $h$, we have (5.5)

$$
\kappa(\kappa-1) g(\mathrm{X}, \mathrm{Z}) \eta(\mathrm{Y}) \eta(\mathrm{T})-\kappa(\kappa-1) \eta(\mathrm{X}) \eta(\mathrm{Y}) \eta(\mathrm{Z}) \eta(\mathrm{T})+\mu(\kappa-1) g(h \mathrm{X}, \mathrm{Z}) \eta(\mathrm{Y}) \eta(\mathrm{T})
$$




$$
\begin{aligned}
& -\kappa(\kappa-1) g(\mathrm{Y}, \phi \mathrm{Z}) g(\phi \mathrm{X}, \mathrm{T})-\kappa g(h \mathrm{X}, \phi \mathrm{Z}) g(\phi h \mathrm{Y}, \mathrm{T})+\kappa g(\mathrm{Z}, \phi h \mathrm{Y}) g(\phi h \mathrm{X}, \mathrm{T}) \\
& +\kappa(\kappa-1) g(\mathrm{Z}, \phi \mathrm{X}) g(\phi \mathrm{Y}, \mathrm{T})+\kappa(\kappa-1) \eta(\mathrm{Y}) \eta(\mathrm{Z}) g(\mathrm{X}, \mathrm{T})-\kappa(\kappa-1) \eta(\mathrm{X}) \eta(\mathrm{Y}) \eta(\mathrm{Z}) \eta(\mathrm{T}) \\
& -\mu(1-\kappa) \eta(\mathrm{Y}) \eta(\mathrm{Z}) g(h \mathrm{X}, \mathrm{T})-2 \mu g(h \mathrm{X}, \phi \mathrm{Y}) g(\phi h \mathrm{Z}, \mathrm{T})-\frac{1}{4 \mathrm{n}}[\mathrm{S}(\mathrm{Y}, h \mathrm{Z}) g(h \mathrm{X}, \mathrm{T}) \\
& -\mathrm{S}(h \mathrm{X}, h \mathrm{Z}) g(\mathrm{Y}, \mathrm{T})+g(\mathrm{Y}, h \mathrm{Z}) \mathrm{S}(h \mathrm{X}, \mathrm{T})-g(h \mathrm{X}, h \mathrm{Z}) \mathrm{S}(\mathrm{Y}, \mathrm{T})+(\kappa-1) \mathrm{S}(\mathrm{Y}, \mathrm{Z}) g(\mathrm{X}, \mathrm{T}) \\
& -(\kappa-1) \mathrm{S}(\mathrm{Y}, \mathrm{Z}) \eta(\mathrm{X}) \eta(\mathrm{T})+\mathrm{S}(h \mathrm{X}, \mathrm{Z}) g(h \mathrm{Y}, \mathrm{T})+(\kappa-1) g(\mathrm{Y}, \mathrm{Z}) \mathrm{S}(\mathrm{X}, \mathrm{T}) \\
& -\kappa(\kappa-1)(\mathrm{n}-1) g(\mathrm{Y}, \mathrm{Z}) \eta(\mathrm{X}) \eta(\mathrm{T})+g(h \mathrm{X}, \mathrm{Z}) \mathrm{S}(h \mathrm{Y}, \mathrm{T})]=0 .
\end{aligned}
$$

Treating $Y=T=\xi$ in (5.5), we get

$$
\begin{aligned}
\mathrm{S}(h \mathrm{X}, h \mathrm{Z})= & \kappa(1-\kappa)(6 \mathrm{n}+4) g(\mathrm{X}, \mathrm{Z})+\kappa(\kappa-1)(6 \mathrm{n}+4) \eta(\mathrm{X}) \eta(\mathrm{Z}) \\
& -\mu(\kappa-1) 4(2 \mathrm{n}+1) g(h \mathrm{X}, \mathrm{Z}) .
\end{aligned}
$$

Plugging $X$ by $h X$ and $Z$ by $h Z$ in (5.6) and applying equations (2.1), (2.7), (2.9), we obtain

$$
\begin{aligned}
\mathrm{S}(\mathrm{X}, \mathrm{Z})= & \kappa(\kappa-1)(6 \mathrm{n}+4) g(\mathrm{X}, \mathrm{Z})+\kappa[2 \mathrm{n}-(\kappa-1)(6 \mathrm{n}+4)] \eta(\mathrm{X}) \eta(\mathrm{Z}) \\
& +4 \mu(\kappa-1)(2 \mathrm{n}+1) g(h \mathrm{X}, \mathrm{Z}) .
\end{aligned}
$$

By virtue of (2.10), (5.7) reduces to

$$
S(X, Z)=B_{1} g(X, Z)+B_{2} \eta(X) \eta(Z),
$$

where

$B_{1}=\frac{\{\kappa(\kappa-1)(6 n+4)[2(n-1)+\mu]-4(2 n+1) \mu(\kappa-1)[2(n-1)-n \mu]\}}{[2(n-1)+\mu]-4(2 n+1) \mu(\kappa-1)\}}$ and

$B_{2}=\frac{\{\kappa[2 n-(\kappa-1)(6 n+4)][2(n-1)+\mu]-4(2 n+1) \mu(\kappa-1)[2(1-n)+n(2 \kappa+\mu)]\}}{\{[2(n-1)+\mu]-4(2 n+1) \mu(\kappa-1)\}}$.

Thus we get the following theorem:

Theorem 5.4. Let $M^{2 n+1}(\phi, \xi, \eta, g)$ be a non-Sasakian $(\kappa, \mu)$-contact metric manifold. If $M$ is $h$ - $m$-projectively semisymmetric then the manifold is an $\eta$-Einstein manifold with constant coefficients.

In similar manner, from proposition 2.1 and Theorem 5.4 we can state the following:

Corollary 5.4. If $M^{2 n+1}$ be a $h$-m-projectively semisymmetric $(\kappa, \mu)$-contact metric manifold then the Ricci operator $Q$ commutes with $\phi$ i.e., $Q \phi=\phi Q$.

\section{6. $\phi$-m-projectively semi-symmetric non-Sasakian $(\kappa, \mu)$-contact metric manifold}

Definition 6.4. A Riemannian manifold $\left(M^{2 n+1}, g\right)$ is said to be $\phi$ - $m$-projectively semisymmetric if it satisfies

$$
W^{*}(X, Y) \cdot \phi=0 .
$$


Let $M$ be a $(2 n+1)$-dimensional $\phi$-m-projectively semisymmetric non-Sasakian $(\kappa, \mu)$-contact metric manifold. The condition $W^{*}(X, Y) \cdot \phi=0$.turns into,

$$
\left(W^{*}(X, Y) \cdot \phi\right) Z=W^{*}(X, Y) \phi Z-\phi W^{*}(X, Y) Z=0,
$$

for any vector fields $X, Y, Z$.

Making use of (1.2) and (2.15) in (6.2), we obtain

$$
\begin{aligned}
& \{(1-\kappa)[g(\phi \mathrm{Y}, \mathrm{Z}) \eta(\mathrm{X})-g(\phi \mathrm{X}, \mathrm{Z}) \eta(\mathrm{Y})]+(1-\mu)[g(\phi h \mathrm{Y}, \mathrm{Z}) \eta(\mathrm{X})-g(\phi h \mathrm{X}, \mathrm{Z}) \eta(\mathrm{Y})]\} \xi \\
& -g(\mathrm{Y}+h \mathrm{Y}, \mathrm{Z})(\phi \mathrm{X}+\phi h \mathrm{X})+g(\mathrm{X}+h \mathrm{X}, \mathrm{Z})(\phi \mathrm{Y}+\phi h \mathrm{Y})-g(\phi \mathrm{Y}+\phi h \mathrm{Y}, \mathrm{Z})(\mathrm{X}+h \mathrm{X}) \\
& +g(\phi \mathrm{X}+\phi h \mathrm{X}, \mathrm{Z})(\mathrm{Y}+h \mathrm{Y})-\eta(\mathrm{Z})\{(1-\kappa)[\eta(\mathrm{X}) \phi \mathrm{Y}-\eta(\mathrm{Y}) \phi \mathrm{X}]+(1-\mu)[\eta(\mathrm{X}) \phi h \mathrm{Y} \\
& -\eta(\mathrm{Y}) \phi h \mathrm{X})]\}-\frac{1}{4 \mathrm{n}}[\mathrm{S}(\mathrm{Y}, \phi \mathrm{Z}) \mathrm{X}-\mathrm{S}(\mathrm{X}, \phi \mathrm{Z}) \mathrm{Y}+g(\mathrm{Y}, \phi \mathrm{Z}) \mathrm{QX}-g(\mathrm{X}, \phi \mathrm{Z}) \mathrm{QY}-\mathrm{S}(\mathrm{Y}, \mathrm{Z}) \phi \mathrm{X} \\
& +\mathrm{S}(\mathrm{X}, \mathrm{Z}) \phi \mathrm{Y}-g(\mathrm{Y}, \mathrm{Z}) \mathrm{Q} \phi \mathrm{X}+g(\mathrm{X}, \mathrm{Z}) \mathrm{Q} \phi \mathrm{Y}]=0 .
\end{aligned}
$$

Now replacing $X$ by $\phi X$ and using equations (2.1), (2.4), (2.5), (2.11) and symmetry property of $h$ in (6.3), we get

$$
\begin{aligned}
& (1-\kappa) g(\mathrm{X}, \mathrm{Z}) \eta(\mathrm{Y}) \xi-(1-\kappa) \eta(\mathrm{X}) \eta(\mathrm{Y}) \eta(\mathrm{Z}) \xi-(1-\mu) g(h \mathrm{X}, \mathrm{Z}) \eta(\mathrm{Y}) \xi+g(\mathrm{Y}, \mathrm{Z}) \mathrm{X} \\
& -g(\mathrm{Y}, \mathrm{Z}) \eta(\mathrm{X}) \xi-g(\mathrm{Y}, \mathrm{Z}) h \mathrm{X}+g(h \mathrm{Y}, \mathrm{Z}) \mathrm{X}-g(h \mathrm{Y}, \mathrm{Z}) \eta(\mathrm{X}) \xi-g(h \mathrm{Y}, \mathrm{Z}) h \mathrm{X}+g(\phi \mathrm{X}, \mathrm{Z}) \phi \mathrm{Y} \\
& +g(\phi \mathrm{X}, \mathrm{Z}) \phi h \mathrm{Y}+g(h \phi \mathrm{X}, \mathrm{Z}) \phi \mathrm{Y}+g(h \phi \mathrm{X}, \mathrm{Z}) \phi h \mathrm{Y}-g(\phi \mathrm{Y}, \mathrm{Z}) \phi \mathrm{X}-g(\phi \mathrm{Y}, \mathrm{Z}) h \phi \mathrm{X} \\
& -g(\phi h \mathrm{Y}, \mathrm{Z}) \phi \mathrm{X}-g(\phi h \mathrm{Y}, \mathrm{Z}) h \phi \mathrm{X}-g(\mathrm{X}, \mathrm{Z}) \mathrm{Y}+\eta(\mathrm{X}) \eta(\mathrm{Z}) \mathrm{Y}+g(h \mathrm{X}, \mathrm{Z}) \mathrm{Y}-g(\mathrm{X}, \mathrm{Z}) h \mathrm{Y} \\
& +\eta(\mathrm{X}) \eta(\mathrm{Z}) h \mathrm{Y}+g(h \mathrm{X}, \mathrm{Z}) h \mathrm{Y}-(1-\kappa) \eta(\mathrm{Y}) \eta(\mathrm{Z}) \mathrm{X}+(1-\kappa) \eta(\mathrm{X}) \eta(\mathrm{Y}) \eta(\mathrm{Z}) \xi \\
& -(1-\mu) \eta(\mathrm{Y}) \eta(\mathrm{Z}) h \mathrm{X}-\frac{1}{4 \mathrm{n}}[\mathrm{S}(\mathrm{Y}, \phi \mathrm{Z}) \phi \mathrm{X}-\mathrm{S}(\mathrm{X}, \mathrm{Z}) \mathrm{Y}+2 \mathrm{n} \kappa \eta(\mathrm{X}) \eta(\mathrm{Z}) \mathrm{Y} \\
& +2(2 \mathrm{n}-2+\mu) g(h \mathrm{X}, \mathrm{Z}) \mathrm{Y}+g(\mathrm{Y}, \phi \mathrm{Z}) \mathrm{Q} \phi \mathrm{X}-g(\mathrm{X}, \mathrm{Z}) \mathrm{QY}+\eta(\mathrm{X}) \eta(\mathrm{Z}) \mathrm{QY}+\mathrm{S}(\mathrm{Y}, \mathrm{Z}) \mathrm{X} \\
& -\mathrm{S}(\mathrm{Y}, \mathrm{Z}) \eta(\mathrm{X}) \xi+\mathrm{S}(\phi \mathrm{X}, \mathrm{Z}) \phi \mathrm{Y}+g(\mathrm{Y}, \mathrm{Z}) \mathrm{QX}-g(\mathrm{Y}, \mathrm{Z}) \phi(\mathrm{X}) \mathrm{Q} \xi+g(\phi \mathrm{X}, \mathrm{Z}) \mathrm{Q} \phi \mathrm{Y}]=0 .
\end{aligned}
$$

Taking inner product of (6.4) with $T$ and using (2.1), (2.9), (2.11), (2.12), we obtain

$$
\begin{aligned}
& (1-\kappa) g(\mathrm{X}, \mathrm{Z}) \eta(\mathrm{Y}) \eta(\mathrm{T})-(1-\kappa) \eta(\mathrm{X}) \eta(\mathrm{Y}) \eta(\mathrm{Z}) \eta(\mathrm{T})-(1-\mu) g(h \mathrm{X}, \mathrm{Z}) \eta(\mathrm{Y}) \eta(\mathrm{T}) \\
& +g(\mathrm{Y}, \mathrm{Z}) g(\mathrm{X}, \mathrm{T})-g(\mathrm{Y}, \mathrm{Z}) \eta(\mathrm{X}) \eta(\mathrm{T})-g(\mathrm{Y}, \mathrm{Z}) g(h \mathrm{X}, \mathrm{T})+g(h \mathrm{Y}, \mathrm{Z}) g(\mathrm{X}, \mathrm{T}) \\
& -g(h \mathrm{Y}, \mathrm{Z}) \eta(\mathrm{X}) \eta(\mathrm{T})-g(h \mathrm{Y}, \mathrm{Z}) g(h \mathrm{X}, \mathrm{T})+g(\phi \mathrm{X}, \mathrm{Z}) g(\phi \mathrm{Y}, \mathrm{T})+g(\phi \mathrm{X}, \mathrm{Z}) g(\phi h \mathrm{Y}, \mathrm{T}) \\
& +g(h \phi \mathrm{X}, \mathrm{Z}) g(\phi \mathrm{Y}, \mathrm{T})+g(h \phi \mathrm{X}, \mathrm{Z}) g(\phi h \mathrm{Y}, \mathrm{T})-g(\phi \mathrm{Y}, \mathrm{Z}) g(\phi \mathrm{X}, \mathrm{T})-g(\phi \mathrm{Y}, \mathrm{Z}) g(h \phi \mathrm{X}, \mathrm{T}) \\
& -g(\phi h \mathrm{Y}, \mathrm{Z}) g(\phi \mathrm{X}, \mathrm{T})-g(\phi h \mathrm{Y}, \mathrm{Z}) g(h \phi \mathrm{X}, \mathrm{T})-g(\mathrm{X}, \mathrm{Z}) g(\mathrm{Y}, \mathrm{T})+\eta(\mathrm{X}) \eta(\mathrm{Z}) g(\mathrm{Y}, \mathrm{T}) \\
& +g(h \mathrm{X}, \mathrm{Z}) g(\mathrm{Y}, \mathrm{T})-g(\mathrm{X}, \mathrm{Z}) g(h \mathrm{Y}, \mathrm{T})+\eta(\mathrm{X}) \eta(\mathrm{Z}) g(h \mathrm{Y}, \mathrm{T})+g(h \mathrm{X}, \mathrm{Z}) g(h \mathrm{Y}, \mathrm{T}) \\
& -(1-\kappa) \eta(\mathrm{Y}) \eta(\mathrm{Z}) g(\mathrm{X}, \mathrm{T})+(1-\kappa) \eta(\mathrm{X}) \eta(\mathrm{Y}) \eta(\mathrm{Z}) \eta(\mathrm{T})-(1-\mu) \eta(\mathrm{Y}) \eta(\mathrm{Z}) g(h \mathrm{X}, \mathrm{W})
\end{aligned}
$$




$$
\begin{aligned}
& -\frac{1}{4 \mathrm{n}}[\mathrm{S}(\mathrm{Y}, \phi \mathrm{Z}) g(\phi \mathrm{X}, \mathrm{T})-\mathrm{S}(\mathrm{X}, \mathrm{Z}) g(\mathrm{Y}, \mathrm{T})+2 \mathrm{n} \kappa \eta(\mathrm{X}) \eta(\mathrm{Z}) g(\mathrm{Y}, \mathrm{T})+2(2 \mathrm{n}-2+\mu) \\
& g(h \mathrm{X}, \mathrm{Z}) g(\mathrm{Y}, \mathrm{T})+g(\mathrm{Y}, \phi \mathrm{Z}) \mathrm{S}(\phi \mathrm{X}, \mathrm{T})-g(\mathrm{X}, \mathrm{Z}) \mathrm{S}(\mathrm{Y}, \mathrm{T})+\eta(\mathrm{X}) \eta(\mathrm{Z}) \mathrm{S}(\mathrm{Y}, \mathrm{T}) \\
& +\mathrm{S}(\mathrm{Y}, \mathrm{Z}) g(\mathrm{X}, \mathrm{T})-\mathrm{S}(\mathrm{Y}, \mathrm{Z}) \eta(\mathrm{X}) \eta(\mathrm{T})-\mathrm{S}(\phi \mathrm{X}, \mathrm{Z}) g(\phi \mathrm{Y}, \mathrm{T})+g(\mathrm{Y}, \mathrm{Z}) \mathrm{S}(\mathrm{X}, \mathrm{T}) \\
& +(\mathrm{n}-1) \kappa g(\mathrm{Y}, \mathrm{Z}) \eta(\mathrm{X}) \eta(\mathrm{T})-g(\phi \mathrm{X}, \mathrm{Z}) \mathrm{S}(\phi \mathrm{Y}, \mathrm{W})]=0 .
\end{aligned}
$$

Treating $Y=T=\xi$ in (6.5) and using (2.1), (2.2), (2.4), (2.5) and (2.11), we get

$$
\begin{aligned}
\mathrm{S}(\mathrm{X}, \mathrm{Z})= & \kappa(6 \mathrm{n}+4) g(\mathrm{X}, \mathrm{Z})+\{-4 \kappa(\mathrm{n}+1)\} \eta(\mathrm{X}) \eta(\mathrm{Z})+\{2(2 \mathrm{n}-2+\mu) \\
& -4 \mu(2 \mathrm{n}+1)\} g(\mathrm{~h} \mathrm{X}, \mathrm{Z}) .
\end{aligned}
$$

Now using (2.10) in (6.6), we have

$$
\mathrm{S}(\mathrm{X}, \mathrm{Z})=\mathrm{B}_{3} g(\mathrm{X}, \mathrm{Z})+\mathrm{B}_{4} \eta(\mathrm{X}) \eta(\mathrm{Z}) .
$$

Where $B_{3}=\frac{\{\kappa(6 n+4)[2(n-1)+\mu]-[2(2(n-1)+\mu)-4 n \mu][2(n-1)-n \mu]\}}{[2 n-2+\mu]-[2(2(n-1)+\mu)-4(2 n+1) \mu]}$

and

$$
B_{4}=\frac{\{-4 \kappa(n+1)[2(n-1)+\mu]-[2(2(n-1)+\mu)-4(2 n+1) \mu][2(1-n)+n(2 \kappa+\mu)]\}}{[2(n-1)+\mu]-[2(2 n-2+\mu)-4(2 n+1) \mu]} .
$$

Thus we have the following theorem:

Theorem 6.5. If $(2 n+1)$-dimensional non-Sasakian $(\kappa, \mu)$-contact metric manifold $M$ is $\phi$ - $m$-projectively semisymmetric then the manifold is an $\eta$-Einstein manifold with constant coefficients.

In similar manner, from proposition 2.1 and Theorem 6.5 we can state the following:

Corollary 6.5. If $M^{2 n+1}$ be a $\phi$-m-projectively semisymmetric $(\kappa, \mu)$-contact metric manifold then the Ricci operator $Q$ commutes with $\phi$ i.e., $Q \phi=\phi Q$.

\section{Acknowledgement:-}

The first author is thankful to UGC for financial support in the form of Rajiv Gandhi National Fellowship (F117.1/2015-16/RGNF-2015-17-SC-KAR-26367).

\section{References:-}

1. Ahmet Yildiz and U. C. De, A classification of $(\kappa, \mu)$-contact metric manifolds, Commun. Korean Math.Soc., 2, 327-339, 2012.

2. Blair D. E, Contact manifolds in Riemannian geometry, Lecture Notes in Math. 509, Springer-Verlag, 1976.

3. D.E. Blair, T. Koufogiorgos and B.J. Papantoniou, Contact metric manifolds satisfying a nullity condition, Israel J. Math. 19, 189-214, 1995.

4. E. Boeckx, A full classification of contact metric $(\kappa, \mu)$-spaces, Illinois J. of Math. 44, 212-219, 2000.

5. E. Boeckx, P. Buecken and L. Vanhecke, $\phi$-symmetric contact metric spaces, Glasg. Math. J. 41(3), 409-416, 1999.

6. Chaubey S. K. and Ojha R. H, On the $M$-projective curvature tensor of a Kenmotsu manifold, Differential Geomerty-Dynamical Systems, 12, 52-60, 2010.

7. Chaubey S. K, Some properties of LP-Sasakian manifolds equipped with $M$-Projective curvature tensor, Bulletin of Mathematical Analysis and Applications, 3(4), 50-58, 2011.

8. U.C. De and A. A. Shaikh, Sasakian manifolds with $C$-Bochner curvature tensor, Indian J. of Math., 41(2), 131$137,1999$.

9. H. Endo, On certain tensor fields on contact metric manifolds II, Publ. Math. Debrecen, 44, 157-166, 1994.

10. A. Ghosh, R. Sharma and J.T. Cho, Contact metric manifolds with $\eta$-parallel torsion tensor, Ann. Glob. Anal. Geom. 34, 287-299, 2008. 
11. Jun J. B. Yildiz A. and De U. C, On $\phi$-recurrent $(\kappa, \mu)$-contact metric manifolds, Bull. Korean Math. Soc. 45, 689-700, 2008.

12. J.S. Kim, M.M. Tripathi and J. Choi, On $C$-Bochner curvature tensor of a contact metric manifold, Bull. Korean Math. Soc., 42(4), 713-724, 2005.

13. O. Kowalski, An explicit classification of 3-dimensional Riemannian spaces satisfying $R(X, Y) \cdot R=0$, Czechoslovak Math. J. 46(121), 3, 427-474, 1996.

14. M. Matsumoto and G. Chuman, On $C$-Bochner curvature tensor, TRU Math., 5, 21-30, 1969.

15. Ojha R. H, A note on the $m$-projective curvature tensor, Indian J. pure Applied Math. 8(12), 1531-1534, 1975.

16. Ojha R. H, On Sasakian manifold, Kyungpook Math. J., 13, 211-215, 1973.

17. D. Perrone, Homogeneous contact Riemannian three-manifolds, Illinois J. Math. 42, 243-258, 1998.

18. Pokhariyal G. P. and Mishra R. S, Curvature tensor and their relativistic significance II, Yokohama Mathematical Journal 19, 97-103, 1971.

19. A. A. Shaikh and K. Kanti Baishya, On $(\kappa, \mu)$-contact metric manifolds, Differential Geometry Dynamical Systems, 8, 253-261, 2006.

20. Z. I. Szabo, Structure theorems on Riemannian spaces satisfying $R(X, Y) \cdot R=0$. I. The local version, J. Differential Geom. 17(4), 531-582, 1982.

21. T. Takahashi, Sasakian $\phi$-symmetric spaces, Tohoku Math. J. (2)29, 1, 91-113, 1977.

22. S. Tanno, Ricci curvatures of contact Riemannian manifolds, Tohoku Math. J. 40, 441-448, 1988.

23. Venkatesha and B. Sumangala, On $m$-projective curvature tensor of a generalized Sasakian space form, Acta Math. Univ. Comenianae, Vol. LXXXII, 2, 209-217, 2013. 